San Jose State University

SJSU ScholarWorks

Master's Theses

Master's Theses and Graduate Research

Fall 2015

\title{
Mitochondrial dysfunction and lipid metabolism play a major role in the lethality caused by developmental alcohol exposure
}

Payam Khodabakhshi

San Jose State University

Follow this and additional works at: https://scholarworks.sjsu.edu/etd_theses

\section{Recommended Citation}

Khodabakhshi, Payam, "Mitochondrial dysfunction and lipid metabolism play a major role in the lethality caused by developmental alcohol exposure" (2015). Master's Theses. 4643.

DOI: https://doi.org/10.31979/etd.xm5e-bp58

https://scholarworks.sjsu.edu/etd_theses/4643

This Thesis is brought to you for free and open access by the Master's Theses and Graduate Research at SJSU ScholarWorks. It has been accepted for inclusion in Master's Theses by an authorized administrator of SJSU ScholarWorks. For more information, please contact scholarworks@sjsu.edu. 


\title{
MITOCHONDRIAL DYSFUNCTION AND LIPID METABOLISM PLAY A MAJOR ROLE IN THE LETHALITY CAUSED BY DEVELOPMENTAL ALCOHOL EXPOSURE
}

\author{
A Thesis \\ Presented to \\ The Faculty of Department of Biological Sciences \\ San José State University \\ In Partial Fulfillment \\ of the Requirements for the Degree \\ Master of Science
}

by

Payam Khodabakhshi

December 2015 
(C) 2015

Payam Khodabakhshi

ALL RIGHTS RESERVED 
The Designated Thesis Committee Approves the Thesis Titled

\section{MITOCHONDRIAL DYSFUNCTION AND LIPID METABOLISM PLAY A MAJOR ROLE IN THE LETHALITY CAUSED BY DEVELOPMENTAL ALCOHOL EXPOSURE \\ by \\ Payam Khodabakhshi}

APPROVED FOR THE DEPARTMENT OF BIOLOGICAL SCIENCES

SAN JOSÉ STATE UNIVERSITY

December 2015
Dr. Rachael French
Department of Biological Sciences
Dr. Katie Wilkinson
Department of Biological Sciences
Dr. Shelley Cargill
Department of Biological Sciences 


\title{
ABSTRACT \\ MITOCHONDRIAL DYSFUNCTION AND LIPID METABOLISM PLAY A MAJOR ROLE IN THE LETHALITY CAUSED BY DEVELOPMENTAL ALCOHOL EXPOSURE
}

\author{
by Payam Khodabakhshi
}

Exposure to ethanol during development causes a variety of physical, developmental, and cognitive abnormalities. In humans, these symptoms are referred to as fetal alcohol syndrome (FAS) or fetal alcohol spectrum disorder (FASD). Previously, we established a Drosophila melanogaster model of FASD and showed that developmental ethanol exposure causes oxidative stress, and that this is a primary cause of the developmental lethality and delay associated with ethanol exposure. In this study, we investigate the role of fatty acid metabolism and lipid accumulation in connection to FASD. Here, we show that developmental ethanol exposure leads to dysregulation of fatty acid metabolism and lipid accumulation. Flies reared in ethanol-containing food had increased fat storage and had increased expression of withered, a lipid metabolism gene. Further, we saw a novel synergistic interaction between ethanol and a long-chain saturated fatty acid (palmitic acid), which strongly indicates that they both have the same molecular target in the cell. Our results show that one of the mechanisms by which ethanol induces oxidative stress is through dysregulation of fatty acid metabolism. These data suggest that dietary changes may prevent some aspects of FASD. 


\section{ACKNOWLEDGEMENTS}

My sincere thanks and deep gratitude first goes to my parents, who have supported me in so many ways throughout my life.

I would like to thank the members of the French lab for their support. Special thanks to Amanda Guevera, Jodie Wu, Patricia Khouderchah, and Sadaf Zaki for their help with my project.

I am especially indebted to Anthony Bortolazzo and Peter Luu, both of whom shared with me their extensive expertise and knowledge since the day I started working in the French lab.

Thanks to Dr. Katie Wilkinson and Dr. Shelley Cargill for their insightful comments to the final manuscript.

And finally, I would like to thank Dr. Rachael French for accepting me as a graduate student and mentoring me. I am very grateful for the education and the research experience I received in her lab. Thank you for your help, advice, and guidance throughout these past two years. 


\section{TABLE OF CONTENTS}

Introduction 1

Fetal Alcohol Spectrum Disorder . . . . . . . . . . . . . 1

Ethanol, Oxidative Stress, and the Role of Lipid Metabolism . . . . . 3

Reactive Oxygen Species . . . . . . . . . . . . . . . 4

Sources of ROS ......................... 5

Reactive Oxygen Species and Oxidative Stress . . . . . . . . . . 6

Fatty Acid Metabolism Pathway . . . . . . . . . . . . 8

Ethanol and Lipid Accumulation . . . . . . . . . . . . . . . . 11

Lipids and Oxidative Stress . . . . . . . . . . . . . . . . 12

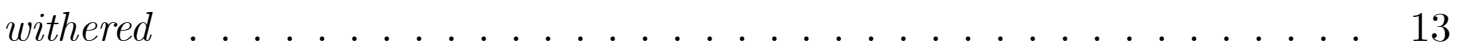

Drosophila as a Model of FAS . . . . . . . . . . . . . . . . 14

Materials and Methods $\quad 15$

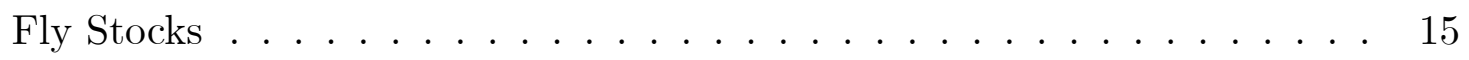

Food Recipes ....................... 15

Lifespan Assay . . . . . . . . . . . . . . . . . . 16

Starvation Assay . . . . . . . . . . . . . . . . 16

Survival Assay . . . . . . . . . . . . . . . . . . 16

Lipid Droplet Staining, Imaging, and Quantifying . . . . . . . . . . . 17

Quantitative RT-PCR .................... 18

$\begin{array}{ll}\text { Results } & 19\end{array}$

Upregulation of Genes Involved in Lipid Metabolism . . . . . . . . . . 19

Lipid Metabolism in Ethanol-Reared Flies and the Role of whd . . . . . . 20

Longevity in whd Mutants . . . . . . . . . . . . . . 22 
Increased Fat Storage in Ethanol-Reared Flies _ . . . . . . . . . 24

Interaction Between Saturated Fatty Acids and Ethanol . . . . . . . 27

Combined Effect of Sugars and Ethanol . . . . . . . . . . . . . 30

whd Gene Expression in Flies Reared in Ethanol Food . . . . . . . . 33

$\begin{array}{ll}\text { Discussion } & 35\end{array}$

Ethanol Induces Oxidative Stress by Disturbing LCFA Metabolism . . . . 35

Ethanol-Reared Flies Have Altered whd Gene Expression . . . . . . . . . 36

Oxidative Stress Effects Longevity . . . . . . . . . . . . . . 36

Increased Fat Storage in EtOH-Reared Flies . . . . . . . . . . . . 37

DAE Induces Starvation Resistance . . . . . . . . . . . . . . 38

Synergistic Effect of EtOH and Fatty Acids . . . . . . . . . . . 38

Future Directions . . . . . . . . . . . . . . . . . . . 39

$\begin{array}{ll}\text { References } & 41\end{array}$ 


\section{LIST OF TABLES}

$1.1 \quad$ Reactive Oxygen Species . . . . . . . . . . . . . . . 5

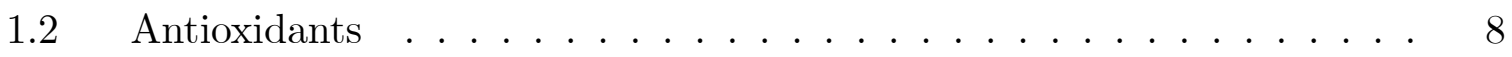

3.1 Microarray Analysis . . . . . . . . . . . . . . 20

3.2 Analysis of Fat Droplets . . . . . . . . . . . . . . 26

3.3 Analysis of Variance for Figure 3.6-A . . . . . . . . . . . . . . 30 


\section{LIST OF FIGURES}

1.1 An Overview of LCFA Metabolism . . . . . . . . . . . . . 10

2.1 Survival Assay Diagram . . . . . . . . . . . . . . . . . . . 17

3.1 Survival of whd Mutants Reared in Ethanol-Containing Food . . . . . 21

3.2 Time to $50 \%$ Eclosion for whd Mutants . . . . . . . . . . . . . . . . 22

$3.3 \quad$ Longevity of Wild-Type Flies Versus $w h d^{1} \ldots \ldots \ldots \ldots$

3.4 Confocal Images of Wild-Type and whd $d^{1}$ Fat Body . . . . . . . . . . 25

3.5 Increased Starvation Resistance of Ethanol-Reared Flies . . . . . . . 27

3.6 Synergistic Effect of Ethanol and Palmitic Acid on Survival . . . . . 29

3.7 Effect of Elevated Sugar on Survival _ . . . . . . . . . . . . . . 32

3.8 whd mRNA Expression . . . . . . . . . . . . . 33 


\section{Introduction}

\section{Fetal Alcohol Spectrum Disorder}

The leading non-genetic cause of mental disability in western societies is the maternal consumption of alcohol during pregnancy (Pulsifer, 1996). This disorder is the result of alcohol's teratogenic effects on the developing fetus and leads to a variety of developmental and cognitive problems, including behavioral changes, slow growth, reduced brain size, and memory problems (Smith, Garic, Berres, \& Flentke, 2014). Developmental alcohol exposure (DAE) also induces craniofacial dysmorphologies, including epicanthal folds, short palpebral fissures, a thin upper lip, and indistinct philtrum (Wattendorf \& Muenke, 2005). These symptoms are referred to as fetal alcohol syndrome (FAS) and sometimes its hypernym, fetal alcohol spectrum disorder (FASD) (Howard et al., 2011).

FAS was first identified in 1973 by Jones and Smith (1973) and since then, diagnostic criteria have been established by the Centers for Disease Control and Prevention (CDC) and partner organizations (CDC, 2014). A detailed guide is available on their website (http://www.cdc.gov/ncbddd/fasd/diagnosis.html), but the following three criteria are the minimum necessary for a diagnosis of FAS, as opposed to FASD:

1. Lack of ridge formation in the upper lip (smooth philtrum), reduced height of the upper lip (thin vermilion border), and reduction in opening of the eye lids (short palpebral fissures)

2. Growth deficits

3. Central nervous system abnormalities

FASD is a non-diagnostic, umbrella term that encapsulates a variety of 
phenotypes of different severities seen in people with developmental alcohol exposure. The most recognizable variety of FASD is FAS (Wattendorf \& Muenke, 2005), which is diagnosed by the criteria given above. In this paper, we discuss FASD. According to a 2013 study done by the U.S. Department of Health and Human Services (HSS), about 9.4\% of pregnant women between the ages of 15-44 reported current alcohol use, $2.3 \%$ reported binge drinking, and $0.4 \%$ reported heavy drinking (HSS, 2014).

Because of alcohol's prevalence in our society, consuming alcohol while unknowingly being pregnant is inevitable for some. Public awareness and educational outreach are therefore very important to inform the general public about the teratogenicity of alcohol. However, even after forty years of these efforts, about $10 \%$ of women drink during pregnancy (CDC, 2012). Some of this persistence could be due to misinformation about moderate drinking during pregnancy, lack of awareness, or unplanned pregnancies, but addiction to alcohol is likely the greatest contributing factor to the continuing incidence of FASD.

FASD not only affects the quality of life of patients and their families, but also puts a financial burden on them and the economy. It is estimated that FASD in the U.S. is responsible for $\$ 4$ billion each year in healthcare costs nationally with the lifetime healthcare cost of a child with FASD estimated around $\$ 2$ million (Lupton, Burd, \& Harwood, 2004). Because simply counseling pregnant women not to drink has been an ineffective way of reducing the incidence of FASD, it becomes important to focus efforts on reducing the detrimental effects of ethanol (EtOH) on the exposed fetus. To accomplish this goal, understanding ethanol's mechanism of action is crucial.

Ethanol does not have a single cellular target, but instead affects multiple 
pathways and proteins. Known targets include ligand-gated ion channels, G-protein-coupled receptors, intracellular signaling proteins (Edenberg \& Foroud, 2013), transcription factors, mitogen-activated protein kinase (MAPK) signaling pathway, dopamine receptors, and insulin-like growth factors (IGFs). There are many potential targets for ethanol and its deleterious effects may come from the disruption of a wide variety of molecular pathways, not all of which are known.

In order to study and have a better understanding of DAE, we have developed a FASD model in Drosophila melanogaster. Using this model, we have previously shown that many of the DAE phenotypes between mammals and flies are similar and that at least one major molecular target (IGF signaling) is conserved between the two species (Logan-Garbisch et al., 2014; McClure, French, \& Heberlein, 2011). The focus of this thesis will be one of those targets, the production of reactive oxygen species (ROS), and its interaction with lipid metabolism, which we also find to be disrupted in ethanol-reared flies.

\section{Ethanol, Oxidative Stress, and the Role of Lipid Metabolism}

Evidence, both direct and indirect, links ethanol exposure with increased production of ROS. Ethanol can increase ROS production through several mechanisms, including increased activity of cellular respiration, decreased antioxidant gene expression, and the direct production of an ethanol-derived radicals (Wu \& Cederbaum, 2003). Chen, Schenker, and Henderson (1997) observed ethanol-induced ROS generation in fetal liver tissue - the major organ for lipid and fatty acid metabolism (Nguyen et al., 2008) — and Heaton, Paiva, Mayer, and Miller (2002) saw a similar effect in the brain. Evidence from our lab demonstrated that ethanol exposure during development increases oxidative stress through the increased production of free radicals as well as the downregulation 
of antioxidant gene expression (Logan-Garbisch et al., 2014). In addition, we observed increased circulating triglyceride levels and lipid storage in larvae reared in ethanol-containing food (Logan-Garbisch et al., 2014), which adds support to the causative relationship between ROS production and increased lipid droplet formation (Liu et al., 2015). Finally, microarray analysis of third-instar larvae reared in ethanol-containing food revealed changes in expression of sixteen genes that are directly involved in lipid metabolism and synthesis, and almost all of these changes are predicted to result in fat accumulation (Logan-Garbisch et al., 2014).

\section{Reactive Oxygen Species}

Reactive oxygen species (ROS) are highly reactive oxygen derivative molecules that fall into two categories: radicals and non-radical ROS (Gupta et al., 2012). Radicals, sometime referred to as free oxygen radicals or free radicals, are byproducts of oxidative phosphorylation and have one or more unpaired electron in their valence shell. Non-radical ROS lack the unpaired electron, but are chemically active and can be converted into ROS. A list of these molecules is shown in Table 1.1. 
Table 1.1. Reactive Oxygen Species

\begin{tabular}{lll}
\hline Name & & Symbol \\
\hline & Radicals & \\
Oxygen (bi-radical) & & $\mathrm{O}_{2}$ \\
Superoxide ion & $\mathrm{O}_{2^{-}}$ \\
Hydroxyl & $\mathrm{OH}$ \\
Nitric oxide & $\mathrm{NO}$ \\
\hline & \\
Hydrogen peroxide & Non-radical \\
Ozone & & $\mathrm{H}_{2} \mathrm{O}_{2}$ \\
Aldehydes & & $\mathrm{O}_{3}$ \\
Singlet oxygen & $\mathrm{HCOR}^{1} \mathrm{O}_{2}$ \\
& & \\
\hline
\end{tabular}

Note. Radical and non-radical oxygen metabolites. Table adapted from Birse et al. (2010).

\section{Sources of ROS}

Reactive oxygen species are produced in the cell through intrinsic and extrinsic sources. Endogenous sources include the mitochondria as a byproduct of respiration, and the immune system as a way to fight off invading pathogens (see below). Exogenous ROS are introduced by air pollutants, radiation, drugs, xenobiotics, and food. Air pollutants, such as cigarette smoke, car exhaust, and industrial pollutants, usually contain nitric oxide derivatives that affect the organism directly (contact with skin) or indirectly (inhale into the lungs). Exposure to radiation, both ionizing and non-ionizing, produces both radicals and non-radical species. $\gamma$-radiation, for example, produces ROS through ionization of water. Non-ionizing radiation (UV-A, UV-B, and UV-C) can produce hydrogen peroxide, superoxide ions, and singlet oxygen. Certain pharmaceutical drugs (e.g. adreamicine and belomycinem) work by inducing ROS production to 
generate protective inflammatory responses for tissue repair, antimicrobial phagocytosis, or apoptosis (Oyinloye, Adenowo, \& Kappo, 2015; Salganik, 2001). Xenobiotics (chemicals, toxins, paraquats) and pathogens (viruses, bacteria) increase ROS levels via different mechanisms: ROS production as a byproduct of xenobiotic metabolism and ROS production as a way to defend against the invading pathogens. Lastly, the majority of the food that is consumed gets oxidized and produces oxidants like peroxides, oxidized fatty acids, and transition metals (Kohen \& Nyska, 2002).

\section{Reactive Oxygen Species and Oxidative Stress}

Not all of the effects of ROS are harmful; phagocytes generate ROS to fight invading pathogens (Oyinloye et al., 2015), and ROS are involved in cell proliferation via the inhibition of tyrosine phosphatase (van Montfort, Congreve, Tisi, Carr, \& Jhoti, 2003), and cell signaling by activating MAPK family of kinases (Stowe \& Camara, 2009). Patients with Chronic Granulomatous Disease, a condition in which cells are unable to form ROS, have difficulty fighting bacterial infections (Zou et al., 2011), which demonstrates the important and beneficial role of ROS in innate immunity. However, excess ROS production leads to toxic effects, including membrane and lipid peroxidation (Kamat, Devasagayam, Priyadarsini, \& Mohan, 2000; Mylonas \& Kouretas, 1999) and DNA damage (Cooke et al., 2003). Lipid peroxidation produces very reactive aldehyde byproducts (such as malonaldehyde and 4-hydroxynonenal) that damage protein molecules (Cabiscol, Tamarit, \& Ros, 2000). Thus, detoxification of ROS is essential for both cellular and organismal survival.

Most ROS are generated by the mitochondrial electron transport chain (Mittal, Siddiqui, Tran, Reddy, \& Malik, 2014). As a result, aerobic life 
forms have evolved defense mechanisms to detoxify excess ROS. Normal cells use a scavenger system to keep ROS production in check (Stowe \& Camara, 2009). If the balance between ROS production and elimination is disrupted and the cell's ROS levels become elevated, the cell is said to be under oxidative stress, which leads to the aforementioned deleterious cellular effects. Exogenous ROS can result from exposure to tobacco, pollutants, viruses, xenobiotics, radiation, and ethanol (Gupta et al., 2012).

Cells use antioxidants, including antioxidant gene products, to fight the deleterious effects of oxidative stress. Antioxidants are compounds that reduce ROS levels through a variety of mechanisms, including removal of radicals and ROS precursors, scavenging ROS, and inhibiting ROS formation. Antioxidants are produced both endogenously and exogenously and fall into two categories: enzymatic and non-enzymatic antioxidants. Enzymatic antioxidants include catalases, glutathione peroxidases (GPxs), and superoxide dismutases (SODs). Non-enzymatic antioxidants come from dietary sources and include molecules such as ascorbic acid (vitamin C), $\alpha$-tocopherol (vitamin E), glutathione (GSH), coenzyme Q, polyphenols, and carotenoids. Table 1.2 contains a list of some of the antioxidants mentioned here. 
Table 1.2. Antioxidants

\begin{tabular}{ll}
\hline Name & Function \\
\hline Ascorbate & $\begin{array}{l}\text { Donate a hydrogen atom to neutralize free } \\
\text { radicals. } \\
\text { Catalase }\end{array}$ \\
$\begin{array}{l}\text { Catalyze the decomposition of } \mathrm{H}_{2} \mathrm{O}_{2} \text { to water } \\
\text { and oxygen. }\end{array}$ \\
Electron acceptor and donor. \\
Glutathione & $\begin{array}{l}\text { Converts } \mathrm{H}_{2} \mathrm{O}_{2} \text { to water. } \\
\text { Reduction of } \mathrm{H}_{2} \mathrm{O}_{2} \text { to water, and lipid } \\
\text { hydroperoxides to their corresponding alcohol. }\end{array}$ \\
Glutathione Peroxidases & $\begin{array}{l}\text { Bind heavy metals via their cysteine residues. } \\
\text { Decomposition of } \mathrm{H}_{2} \mathrm{O}_{2} .\end{array}$ \\
Peroxiredoxins & $\begin{array}{l}\text { Catalyze the dismutation of superoxide } \\
\text { radicals into oxygen and } \mathrm{H}_{2} \mathrm{O}_{2} .\end{array}$ \\
Superoxide Dismutases &
\end{tabular}

Note. A list of antioxidants and their mechanism of action. Table adapted from Massaad and Klann (2011).

Experimental downregulation of expression of the antioxidant genes Glutathione Synthase $(G S)$, Peroxidase $(P x d)$, and rosy $(r y)$ reduces the survival of flies reared in ethanol-containing food, whereas upregulation of those genes has the opposite effect (Logan-Garbisch et al., 2014). These data indicate that at least some of the toxic effects of DAE are due to oxidative stress.

\section{Fatty Acid Metabolism Pathway}

Fatty acids are transported into the cytosol by fatty acid transporter proteins or are taken up by the cell. Once inside the cytosol, medium- and short-chain fatty acids can cross the mitochondrial membranes via diffusion, but long-chain fatty acids (LCFA) require more processing and transporter proteins to do so. LCFAs are converted into acyl-CoA by acyl-CoA synthetase and can either be esterified 
to triglycerides and stored as lipid droplets, or converted to acylcarnitine via carnitine palmitoyl transferase I (CPT1) activity. Once converted, acylcarnitine is transported across inner mitochondria membrane and is converted back to acyl-CoA by carnitine palmitoyl transferase II (CPT2). Acyl-CoA then enters the $\beta$-oxidation cycle where acetyl-CoA, NADH, and $\mathrm{FADH}_{2}$ are produced. Further downstream, acetyl-CoA helps generate energy in the form of ATP by entering the electron transport chain. Figure 1.1 depicts the LCFA part of this pathway. 


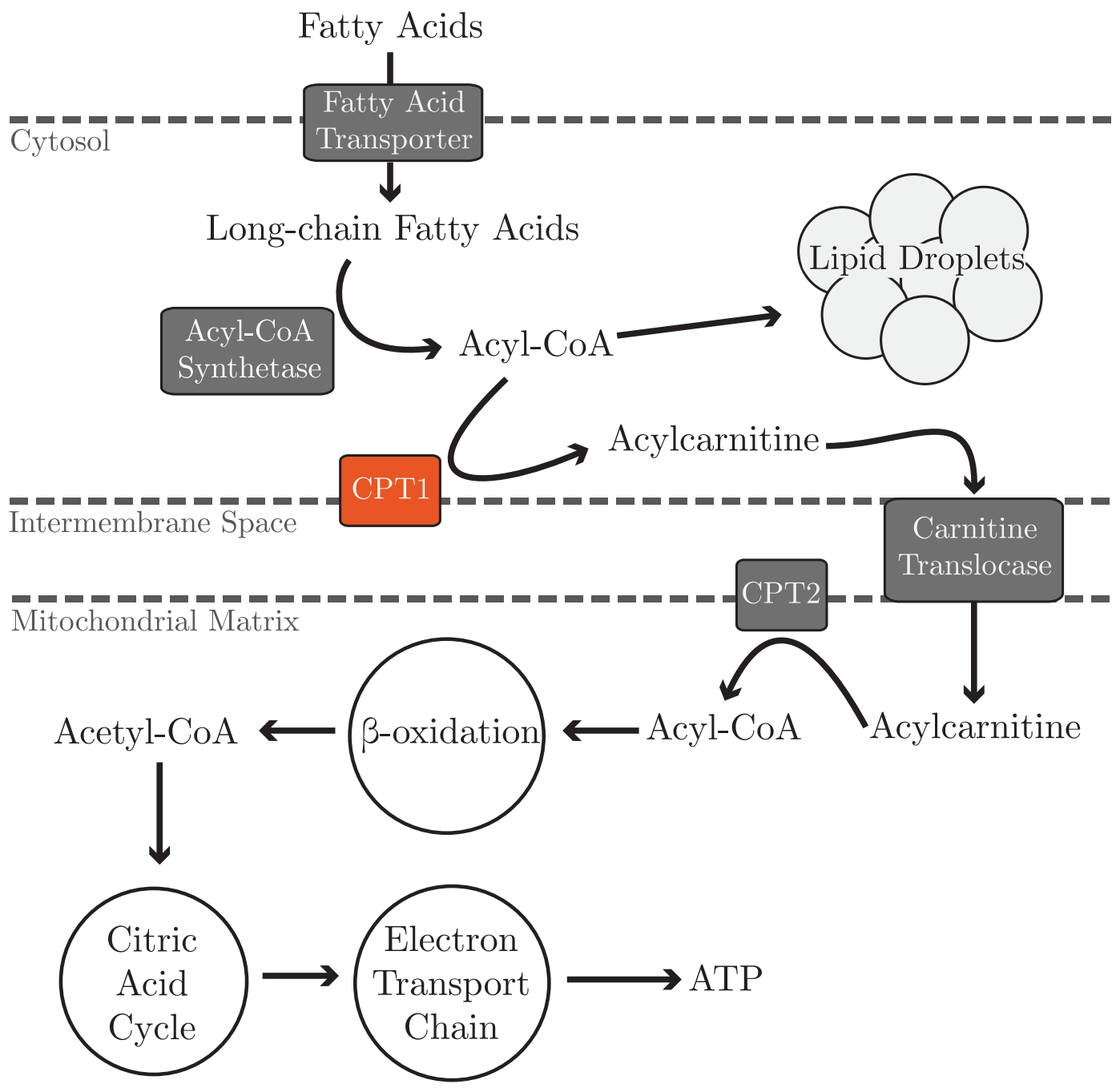

Figure 1.1. An overview of LCFA metabolism. Once inside the cytosol, LCFA are converted to acyl-CoA via acyl-CoA synthase and are stored as lipid droplets or are converted to acylcarnitine via CPT1. Carnitine translocase then transports acylcarnitine to the mitochondrial matrix where CPT2 converts it back to acyl-CoA to be used in $\beta$-oxidation. 


\section{Ethanol and Lipid Accumulation}

Drosophila larvae reared in ethanol-containing food have increased circulating triglyceride levels, as well as increased lipid storage, which is demonstrated by the increased size of the larval fat body (Logan-Garbisch et al., 2014). We have previously shown that Drosophila insulin-like peptides (DILP) signaling is reduced by $75 \%$ in ethanol-reared larvae (McClure et al., 2011), and the obesity phenotype observed in our larvae may be due to reduced DILP expression. Adult Drosophila that have their insulin-producing cells (IPC) removed also display increased circulating and stored lipids (Broughton et al., 2005; Hwangbo, Gershman, Tu, Palmer, \& Tatar, 2004; Pospisilik et al., 2010; Varghese, Lim, \& Cohen, 2010). Fatty acid accumulation causes oxidative stress, cellular toxicity, and can lead to apoptosis (Listenberger et al., 2003). Similarly, obesity in mammals is associated with oxidative stress (Fernández-Sánchez et al., 2011; Furukawa et al., 2004).

Lipid accumulation is known to result in neuronal death during development. Tay-Sachs disease, a developmental neurodegenerative disorder, is caused by a mutation in the lysosomal protein hexosaminidase A (HEXA). This mutation leads to the build-up of the sphingolipid GM2 ganglioside in the brain (Mahuran, 1999). Many nervous system disorders, including Alzheimer's disease, Parkinson's disease, Neiman-Pick disease (NPD), and schizophrenia are associated with dysregulation of lipid metabolism, often in conjunction with oxidative stress (Adibhatla \& Hatcher, 2007). Of particular interest for this work is NPD, as we found a mutation in one of the Drosophila homologs in our screen (Lafler et al., in preparation) and our microarray data show a decrease in

acid Sphingomyelase (aSMase) expression in ethanol-reared larvae (Table 3.1). 
All three forms of NPD are associated with severely reduced levels of aSMase, which results in lysosomal accumulation of sphingolipids. In mouse models, replacement of aSMase activity was effective as a treatment for type B NPD, whereas inhibition of glycosphingolipid synthesis prolonged life in a mouse model

of type C NPD (Miranda et al., 2000; Mukherjee \& Maxfield, 2004; Vance, 2006). This is similar to the increased lifespan observed in flies with a null mutation of withered reared in ethanol-containing food (see Figure 3.3).

\section{Lipids and Oxidative Stress}

We have shown that ethanol exposure during development induces oxidative stress and alters expression of antioxidant genes (Logan-Garbisch et al., 2014). These genes produce compounds that reduce ROS levels through removal of excess radicals and ROS precursors, and by inhibiting ROS formation. One of those genes is withered, which encodes the Drosophila homolog of carnitine palmitoyl transferase I, an enzyme on the outer mitochondrial matrix that facilitates the transfer of long-chain fatty acids into the mitochondria (Strub et al., 2008). CPT1 deficiency in mammals causes shunting of long-chain fatty acids away from the mitochondria and leads to elevated free fatty acid levels (Rasmussen et al., 2002).

Strub et al. (2008) observed that withered mutants are sensitive to oxidative stress induced by paraquat and heavy metals. Similarly, reducing withered activity sensitizes animals to ethanol toxicity (Logan-Garbisch et al., 2014). Ethanol is known to increase ROS production (Chen et al., 1997; Heaton et al., 2002; Ramachandran et al., 2003), thus the sensitivity observed may be the result of oxidative stress overwhelming the cell's antioxidant system and its ability to neutralize ROS. 
Finally, Liu et al. (2015) observed glial-specific lipid droplet (LD) accumulation when they overexpressed c-Jun-N-terminal Kinase (JNK) via prolonged increase in ROS level. JNK pathway activates its own inhibitor, JNK phosphatase, which is suppressed by induction of ROS. The LD accumulation and neurodegeneration were alleviated once ROS levels were reduced (Liu et al., 2015). Clearly, oxidative stress plays a role in fatty acid metabolism and fat storage. The disruption of lipid metabolism by ethanol-induced oxidative stress will be the main focus of this paper and we present our data and findings in Chapter 3.

\section{withered}

In a forward genetic screen for mutations altering survival and development time of flies reared in ethanol-containing food (Lafler et al., in preparation), we identified two independent alleles of the gene withered ( $w h d^{165}$ and $\left.w h d^{896}\right)$. withered (whd) encodes the Drosophila homolog of carnitine palmitoyl transferase I (Strub et al., 2008), a member of the acyltransferase family of proteins. Mammals have three CPT-encoding genes, while the fly genome contains only one (Price et al., 2010).

CPT1 is located in the outer mitochondrial membrane and catalyzes the transfer of the acyl groups of long-chain fatty acyl-CoA to carnitine, making it an essential enzyme for $\beta$-oxidation of long-chain fatty acids (Eaton, 2002; Kerner \& Hoppel, 2000). It is strongly inhibited by malonyl-CoA, which serves as a regulatory mechanism based on cellular fatty acid and glucose availability (Gobin et al., 2003). In mammals, CPT1 has three isoforms, skeletal muscle, hepatic, and brain (Gobin et al., 2003), and its deficiency leads to elevation of free fatty acid levels (hyperlipidemia) due to the shunting of long-chain fatty acids 
away from the mitochondria (Rasmussen et al., 2002). This disruption is more apparent in individuals who are fasting, during which their body uses fats to produce energy, but due to the individual's inability to breakdown LCFA, they can suffer hypoglycemia (Olpin et al., 2001) and are at risk for liver failure, coma, and sudden death (NIH, 2014). CPT1 deficiency reduces the antioxidant properties of carnitine (Juliet Arockia Rani \& Panneerselvam, 2001), which may result in increased ROS production.

\section{Drosophila as a Model of FAS}

Drosophila melanogaster is well-established model organism for studying human diseases. Their conserved biological properties (Pandey \& Nichols, 2011), high reproductive rate, short generation time, low cost, the availability of multiple genetic tools (P elements, CRISPR/Cas9, GAL4-UAS system, flybase.org), and a completely sequenced and annotated genome (Adams et al., 2000) allows researchers to perform large-scale screens that are otherwise difficult or not feasible in other model organisms. We have established Drosophila as a robust model for DAE and have shown that developmental delay and lethality induced by DAE are due to oxidative stress combined with reduced expression of insulin-like peptides and their receptor (Logan-Garbisch et al., 2014; McClure et al., 2011).

Here we provide additional evidence that DAE causes dysregulation of fatty acid metabolism and that this causes oxidative stress and increased developmental lethality. We characterize several alleles of withered and show that lipid metabolism dysregulation leads to increased fat storage. Finally, we show that feeding flies a high-fat diet and ethanol results in a synergistic effect on viability, indicating that the two treatments have the same molecular target. 


\section{Materials and Methods}

\section{Fly Stocks}

We used our standard laboratory background, $w^{1118}$; Wild-Type Berlin $(w ;$ WTB), and obtained the following two strains from the Bloomington Drosophila Stock Center (Bloomington, Indiana): whd $d^{1}$, and $w^{1118} ;$ whd $d^{K G 01596}$.

$w h d^{K G 01596}$ is a partial loss-of-function mutation of the withered gene (FlyBase, 2015) caused by a 15 kilobase transposon insertion, while $w h d^{1}$ is a the null allele caused by a 16 bp deletion that results in a frameshift and the loss of the catalytic C-terminal portion of the CPT1 protein (Strub et al., 2008).

\section{Food Recipes}

Unless otherwise indicated, flies were reared and maintained on standard cornmeal/molasses medium. Modifications used in this thesis are described below.

- 5\% Palmitic Acid (PA) Food: $2.4 \mathrm{~g}$ PA per $50 \mathrm{~mL}$ of standard fly food

- 15\% PA Food: 8.5 g PA per $50 \mathrm{~mL}$ of standard fly food

- 30\% PA Food: 21.45 g PA per $50 \mathrm{~mL}$ of standard fly food

- 8\% Sugar Food: 4.25 g sucrose per $50 \mathrm{~mL}$ of standard fly food

- 5\% Ethanol (EtOH) Food: $2.5 \mathrm{~mL}$ EtOH per $47.5 \mathrm{~mL}$ conditioned food (control, PA, or Sugar)

- 7\% EtOH Food: $3.5 \mathrm{~mL}$ EtOH per $46.5 \mathrm{~mL}$ conditioned food (control, PA or Sugar) 


\section{Lifespan Assay}

Lifespan assays were conducted by collecting age- and phenotype-matched flies and putting them in standard food vials (20 flies per vial). Surviving flies were counted each day. Flies were transferred to new food vials every other day to prevent bacterial growth.

\section{Starvation Assay}

$w$; WTB flies were reared in control medium or food containing $7 \%$ ethanol. After eclosion, 0- to 2-day-old adult flies were transferred to vials containing either standard corn meal/molasses medium (control conditions) or 1\% agarose in water (starvation conditions). Flies were transferred to new vials daily for 6 days (the time at which all starved flies had died), and the surviving flies were counted.

\section{Survival Assay}

Egg collections were taken for 16-20 hours on Petri dishes containing standard fly food. One hundred eggs were then transferred to vials containing either ethanol-containing food, high-fat food, or control food, and, for ethanol exposure experiments, placed in a 5-8\% ethanol bath (experimental conditions; ethanol concentration matches the concentration in the food) or water bath (control conditions). The ethanol bath ensures that developing animals are exposed to ethanol during their entire development, which continues for another 10-16 days. The number of newly eclosed adult flies was counted daily between days 9 and 21 after egg-laying, and these data were used to generate cumulative eclosion rate plots, a direct measurement of egg-to-adult survival, and the time to $50 \%$ of total eclosion. Time to $50 \%$ eclosion was calculated by linear interpolation. 

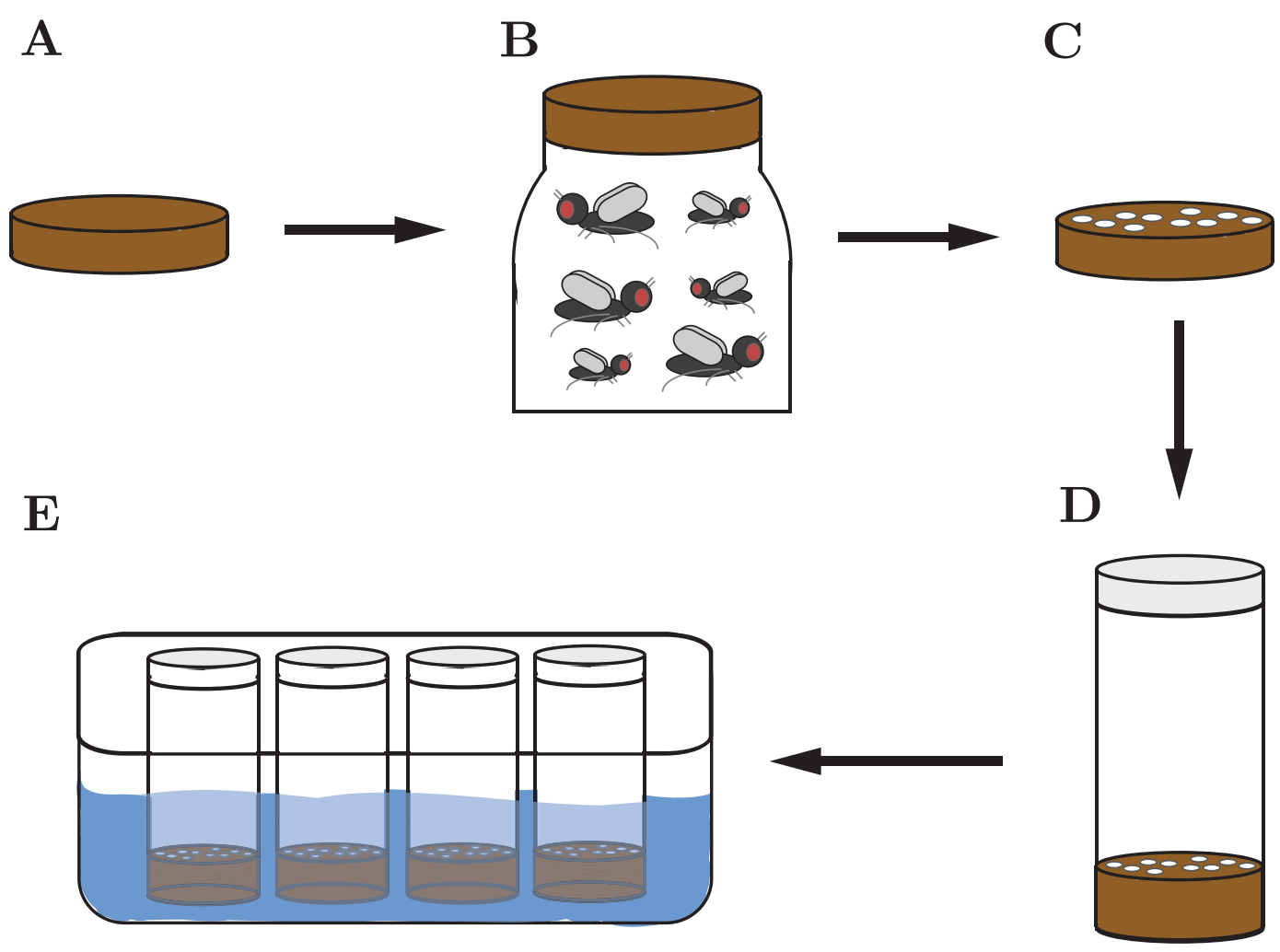

Figure 2.1. Survival assay diagram. (A) Food plates with standard fly food are prepared. (B) Female and male flies (2:1 ratio) are placed inside a bottle to mate and lay eggs. (C) After 24 hours, the food plates are removed and 100 eggs are transferred onto (D) vials containing either experimental food or control food $(n=4)$. (E) Based on the condition of the vials, they are placed inside their respective water or 5-8\% ethanol-containing water baths.

\section{Lipid Droplet Staining, Imaging, and Quantifying}

Larvae were dissected in PBS and fixed in 4\% paraformaldehyde in PBS for 30 min at room temperature. Tissues were then rinsed twice with $1 \times$ PBS, and incubated for $30 \mathrm{~min}$ in a 1:1000 of 0.05\% Nile Red (Sigma, St. Louis, MO). Stained samples were mounted in $75 \%$ glycerol for confocal microscopy analysis. All images were collected on a Zeiss LSM 700 confocal microscope at 100× or $200 \times$ magnification. Lipid droplets were quantified using ImageJ imaging processing program (National Institutes of Health). Color threshold was set 
using the red channel. Droplet counts, total particle area, and percent area were calculated using the Analyze Particle function.

\section{Quantitative RT-PCR}

For quantitative reverse-transcriptase-mediated PCR (qRT-PCR), third instar larvae were snap-frozen on dry ice. Total RNA was extracted using Trizol reagent (Life Technologies, Carlsbad, CA) according to the manufacturer's instructions, resuspended in RNase-free water, and stored at $-80^{\circ} \mathrm{C}$ until use. Total RNA $(2 \mu \mathrm{g})$ was reverse-transcribed using the High-Capacity RNA-to-cDNA Kit (Applied Biosystems, Carlsbad, CA) according to the manufacturer's instructions.

cDNA was analyzed by quantitative real-time PCR using the Applied Biosystems 7300 Real-Time PCR System (Applied Biosystems). The rp49 transcript levels were used as an endogenous normalization control for RNA samples, and relative mRNA abundance was calculated using the comparative $\Delta$ Ct method (Schmittgen and Livak 2008). Each sample was analyzed in triplicate. As negative control, we used DNase-treated nonreverse-transcribed mRNA samples; no significant amplification was observed in these samples. 


\section{Results}

\section{Upregulation of Genes Involved in Lipid Metabolism}

In addition to the identification of two ethanol-sensitive whd alleles, our microarray analysis of ethanol-reared larva revealed changes in the expression of sixteen genes that are directly involved in fatty acid metabolism (Table 3.1), and almost all of these changes are predicted to result in fatty acid accumulation (Logan-Garbisch et al., 2014). These genes include eight genes encoding triglyceride lipases, all of which are reduced by at least five-fold in ethanol-reared larvae and, in one case, by more than 200-fold.

The two genes demonstrating the greatest downregulation in ethanol-reared larvae both encode triglyceride lipases (CG6277, 216-fold; CG6283, 163-fold), and three of the top 11 most downregulated genes fall into this cluster. Additionally, we identified a cluster of downregulated genes encoding glucosylceramidases. Glucosylceramidases catalyze the release of D-glucose and N-acylsphingosine, a free ceramide, from D-glucosyl-N-acylsphingosine. In mammals, glucosylceramidase deficiency leads to lipid accumulation and is the cause of the human neurological disorder Gaucher disease (Logan-Garbisch et al., 2014). Two additional genes (GlcT-1 and aSMase) are altered in ways that would be similarly predicted to decrease free ceramide levels.

Finally, we identified an allele of the gene CG3790, which is predicted to encode the Drosophila carnitine transporter (CT), a protein that catalyzes the movement of acylcarnitine across the inner mitochondrial membrane. This allele, like $w h d^{165}$ and $w h d^{896}$, results in increased developmental delay when reared in ethanol-containing food. 
Table 3.1. Microarray Analysis

\begin{tabular}{|l|c|}
\hline Gene & $\log _{2}$ \\
\hline aSMase & -3.31 \\
\hline ATPCL & -3.05 \\
\hline Gpo-1 & +1.68 \\
\hline CG17191, & \\
CG17192, CG6271, & $-2.35-$ \\
CG6277, CG6283, & -7.75 \\
CG6295, CG8093 & \\
\hline
\end{tabular}

\begin{tabular}{|l|c|}
\hline Gene & $\log _{2}$ \\
\hline Gpdh & +2.04 \\
\hline GlcT-1 & +2.00 \\
\hline Acox57D-d & +1.96 \\
\hline & \\
CG31148, & $-2.68-$ \\
CG31414, CG33090 & -3.46 \\
& \\
\hline
\end{tabular}

Note. Microarray analysis comparing mRNA from ethanol-reared larvae with control larvae. Ethanol-reared wild-type larva show altered expression of genes involved in lipid metabolism.

\section{Lipid Metabolism in Ethanol-Reared Flies and the Role of whd}

We previously carried out a genetic screen to identify mutations that showed altered survival or development time when reared in ethanol-containing food (Lafler et al., in preparation). From this screen, we recovered two alleles of whd and we designated these alleles $w h d^{165}$ and $w h d^{896}$. Both of these alleles resulted in increased developmental delay when reared in ethanol-containing medium. $w h d^{165}$ also showed sensitivity to developmental lethality caused by exposure to ethanol (Logan-Garbisch et al., 2014). To confirm these results, we tested the survival and development time of two additional alleles of whd ( $w h d^{1}$ and $\left.w h d^{K G 01596}\right)$.

Flies that are fed ethanol-containing food show increased developmental mortality, as shown in Figure 3.1. In this experiment, $73 \%$ of control flies reared in ethanol-containing food survived to eclosion. By comparison, $w h d^{1}$, a null mutation of withered (Strub et al., 2008) is exquisitely sensitive to ethanol, exhibiting only $10 \%$ survival to adulthood when reared in ethanol-containing food (Figure 3.1). Similarly, the partial loss-of-function mutation, $w h d^{K G 01596}$, 
shows a $49 \%$ survival on ethanol, which is a significant increase in mortality compared with control animals.

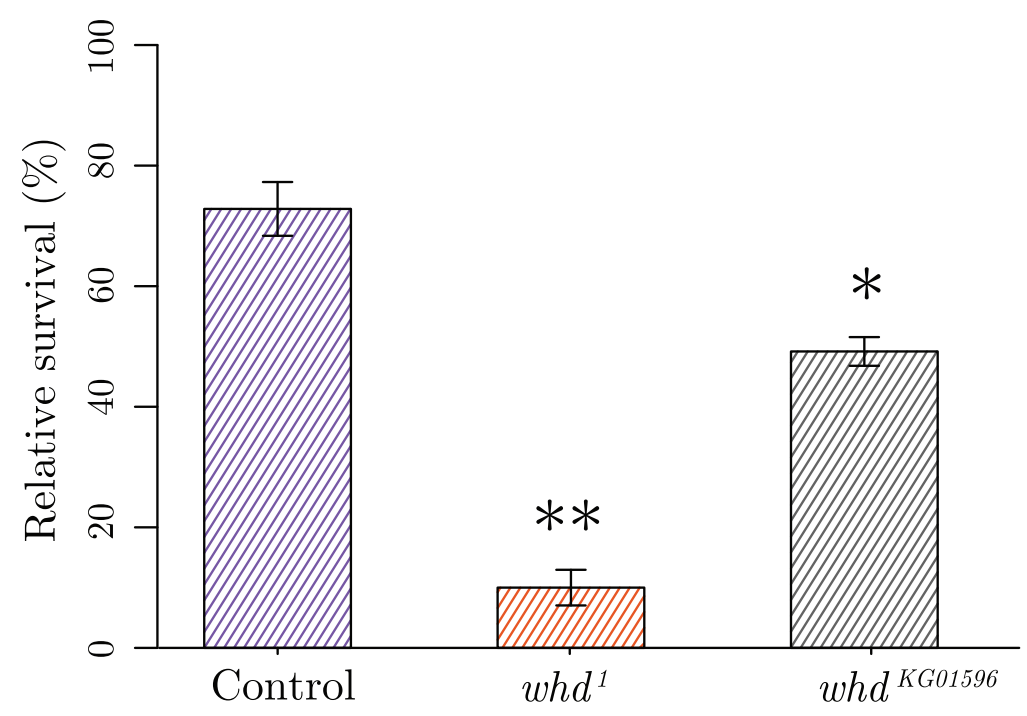

Figure 3.1. Relative survival of whd mutants reared in ethanol-containing food. Survival has been normalized to age- and genotype-matched control flies reared in control food. Error bars represent standard error of the mean $(S E M) . n=4$. ${ }^{*} p<0.05,{ }^{* *} p<0.01$ (One-way ANOVA with Tukey's HSD post hoc analysis).

These data suggest a direct link between ethanol exposure and disruption of lipid metabolism. In addition, we found that $w h d^{1}$ and $w h d^{K G 01596}$ show a significant increase in developmental delay when reared in ethanol-containing food. Figure 3.2 shows the midpoint of eclosion (the point at which $50 \%$ of the flies in a given experiment had eclosed). Wild-type flies on control food reach $50 \%$ eclosion at $10.4 \pm 0.1$ days, and at $13.2 \pm 0.14$ days on $7 \%$ ethanol-containing food. Time to $50 \%$ eclosion for $w h d^{1}$ flies on control food is identical to controls (10.4 \pm 0.03 days) and $13.7 \pm 0.3$ days on ethanol-containing food, a non-significant increase trend in development time. Taken with the previous observation that whd alleles are sensitive to oxidative stress, our data suggest that ethanol causes oxidative stress during development by disrupting fatty acid metabolism. 

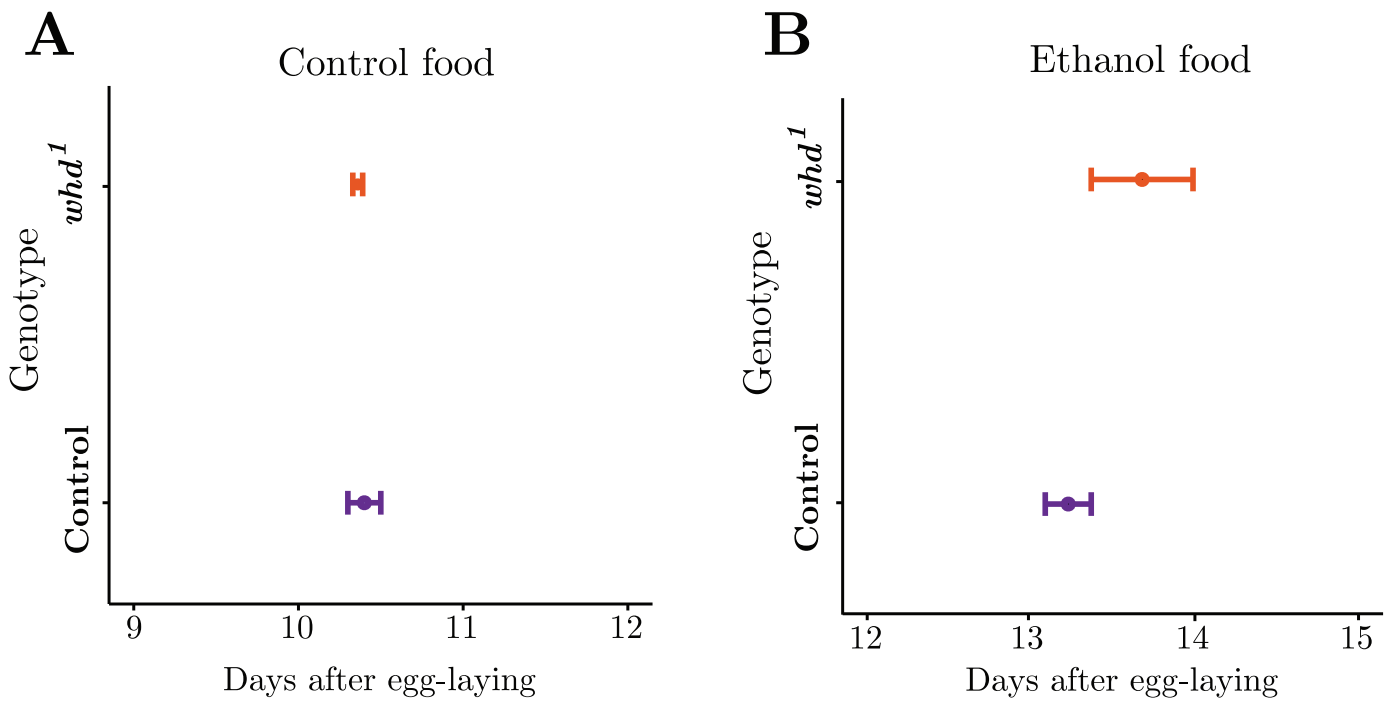

Figure 3.2. Time to $50 \%$ eclosion for whd mutants. Wild-type flies and $w h d^{1}$ mutants were reared in 0\% (A) or 7\% (B) ethanol-containing food. The mean of each condition's midpoint (50\%) eclosion is plotted. Error bars represent $\pm S E M$. $n=4$. Not significant (one-way ANOVA with Tukey's HSD post hoc).

\section{Longevity in whd Mutants}

Reactive oxygen species are a primary cause of aging and reduced lifespan (Kregel \& Zhang, 2007; Valko et al., 2007). Because ethanol-reared flies have an increased level of oxidative stress (Logan-Garbisch et al., 2014), we tested whether the longevity of these flies was affected. We predicted that ethanol-reared flies would have a shorter lifespan due to increased oxidative stress. We reared wild-type and $w h d^{1}$ flies in control food or food containing $7 \%$ ethanol, and counted the surviving flies each day. Both wild-type and $w h d^{1}$ mutant flies, regardless of developmental exposure to ethanol, have a maximum lifespan of approximately eighty days. Further, ethanol-rearing does not significantly impact the median lifespan of control flies. Wild-type flies reared in control food had a median lifespan of $62.8 \pm 3.6$ days, compared with $63.33 \pm 1.2$ days after rearing in ethanol-containing food (Figure 3.3). However, ethanol had 
an interesting interaction with mutation of whd. The average lifespan of $w h d^{1}$ flies reared in control food is much shorter than that of control flies (50.8 \pm 2.2 days). This result is expected, because mutation of whd leads to increased oxidative stress (Strub et al., 2008), which has frequently been shown to reduce lifespan (Sykiotis \& Bohmann, 2008). Intriguingly, and contrary to our expectations, rearing flies in ethanol appears to reverse this effect on lifespan. $w h d^{1}$ flies had an average lifespan of $66.3 \pm 1.9$ days, slightly longer than that of wild-type files in the same condition.

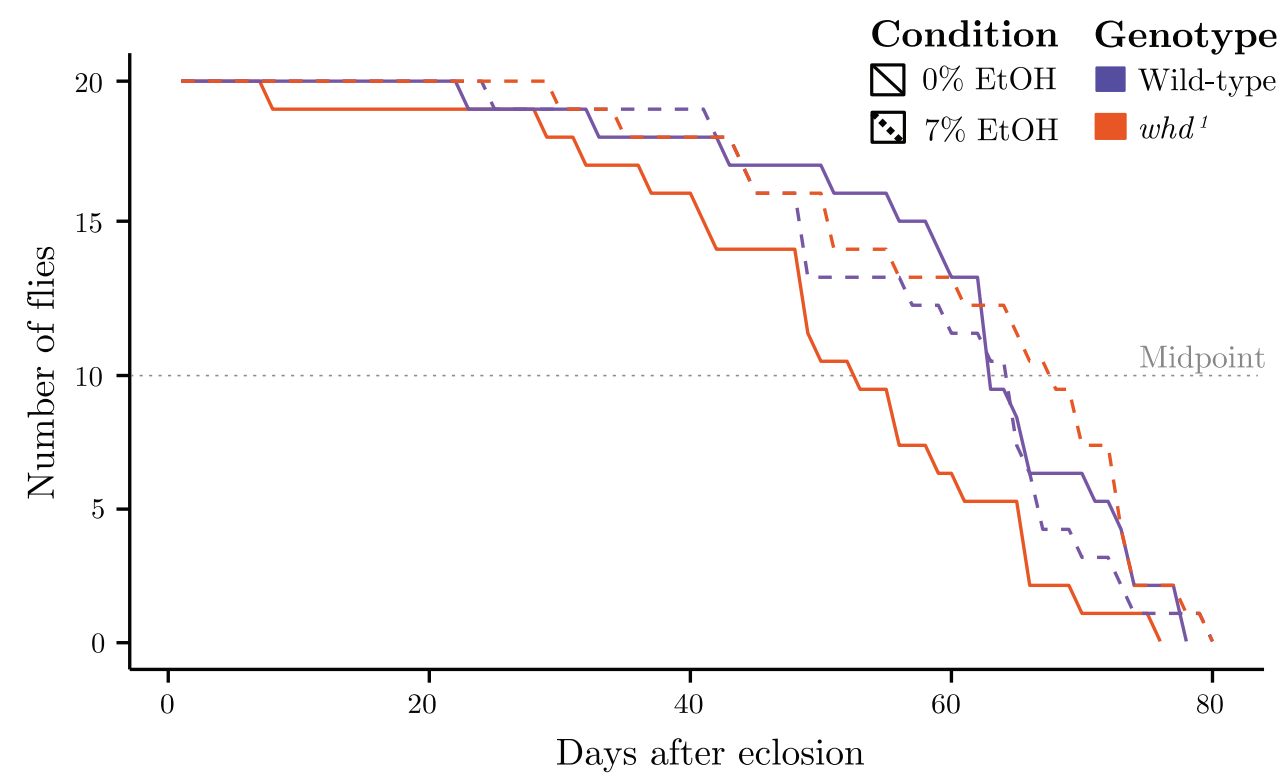

Figure 3.3. Longevity assay. Control flies and $w h d^{1}$ mutants were reared in standard fly food ( $0 \% \mathrm{EtOH}$ ) or ethanol-containing food ( $7 \% \mathrm{EtOH}$ ). Surviving flies were counted every day and were transferred to fresh vials every other day $n=3$-4. Ethanol-rearing had no effect on the lifespans of wild-type flies, while $w h d^{1}$ flies that were reared in ethanol-containing food showed increased longevity compared to flies of the same genotype reared in control food.

These results are surprising because $w h d^{1}$ flies are sensitive to oxidative stress (Strub et al., 2008), and ethanol-rearing leads to increased oxidative stress (Logan-Garbisch et al., 2014). We therefore predicted that ethanol-rearing would reduce the average lifespan of $w h d^{1}$ mutants. We hypothesize that this 
result is due to the upregulation or activation of other antioxidant genes by the small number of flies that survive being reared in ethanol-containing food (approximately 10\%, see Figure 3.3), resulting in a subset of $w h d^{1}$ mutant flies in which ethanol-rearing leads to increased resistance to oxidative stress. This hypothesis could be tested by examining the expression of antioxidant genes such as Catalase (Cat), Peroxidase (Pxd), and superoxide dismutase 1 (sod1) in ethanol-reared whd flies.

\section{Increased Fat Storage in Ethanol-Reared Flies}

The gene expression changes described in Table 3.1 would be expected to result in lipid accumulation. Further, since whd flies are both ethanol-sensitive and have a defect in lipid metabolism, we used Nile Red staining and confocal microscopy to examine lipid storage in whd mutants as well as ethanol-reared wild-type flies. We examined the fat bodies of ethanol-reared wild-type larvae and $w h d^{1}$ larvae reared in control food using Nile Red staining and confocal microscopy (Table 3.2). In both ethanol-reared flies and whd mutants, we observed an increase in the size of lipid droplets, indicating a significant increase in stored fat under both conditions (Figure 3.4). In addition to increased size, lipid droplets in wild-type flies reared in ethanol are increased in numbers. 

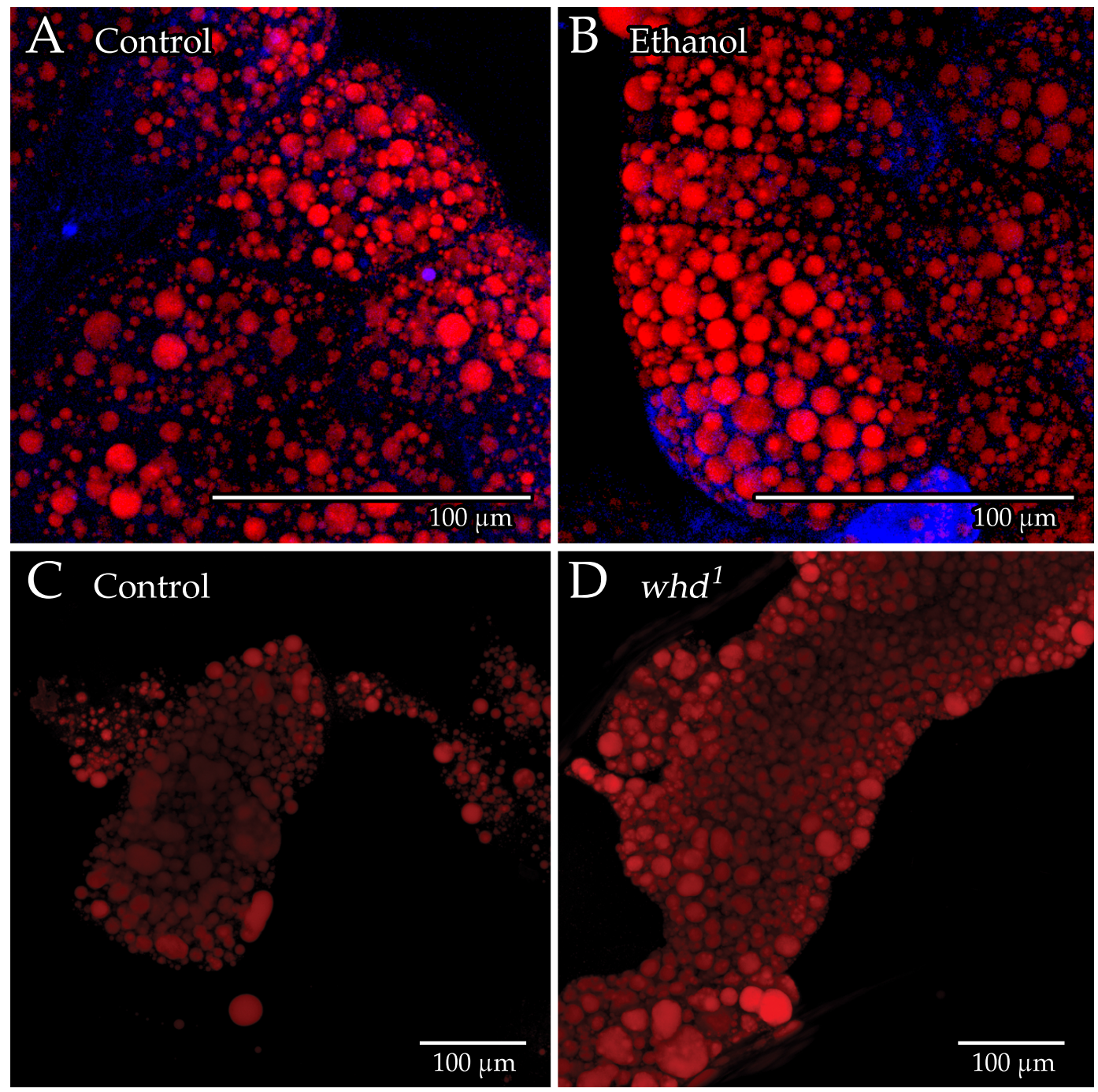

Figure 3.4. Ethanol-reared wild-type flies and $w h d^{1}$ flies show increased lipid storage. (A \& C) Confocal reconstructions of Nile Red-stained fat bodies dissected from wandering third instar wild-type larvae reared in control food. (B) Confocal reconstruction of a Nile Red-stained fat body dissected from an ethanol-reared wandering third instar wild-type larva. (D) Confocal reconstruction of a Nile Red-stained fat body dissected from a wandering third instar $w h d^{1}$ larva reared in control food. Note the increased diameter of fat droplets in (B) and (D) relative to the control fat body shown in (A) and (C). Images A and B are at 200× magnification and images $\mathrm{C}$ and D are $100 \times$ magnification. 
Table 3.2. Analysis of Fat Droplets

\begin{tabular}{lccc}
\hline Sample & Count & Total Particle Area & Percent Area \\
\hline (A) Control & 2,888 & 152,133 & 27.191 \\
(B) Ethanol & 4,250 & 257,096 & 45.951 \\
& & & \\
(C) Control & 443 & 220,709 & 21.048 \\
(D) $w h d^{1}$ & 297 & 403,641 & 38.494 \\
\hline
\end{tabular}

Note. Analysis of fat droplets using ImageJ. Fat droplets of wild-type flies reared in ethanol (B) and withered mutants (D) are increased in size relative to their control. The number of fat droplets in wild-type flies reared in ethanol (B) is also increased relative to its control (A).

This increase in the number of fat droplets is likely caused by the mutant's inability to breakdown long-chain fatty acids, where instead of being metabolized, the fatty acids are converted into fat droplets (see Figure 1.1). The increase is also similar to what we saw when we reared a wild-type larva in ethanol-food (Logan-Garbisch et al., 2014), confirming ethanol's adverse involvement in lipid metabolism, which causes fat accumulation.

We further examined lipid accumulation by starving adult flies that had been reared in ethanol-containing food. Flies were provided with medium composed of $1 \%$ agarose, which provides a source of water but no nutrition, and surviving flies were counted each day. Ethanol-reared flies lived longer, with a median survival time of 3.8 days compared with 2.3 days for control flies (Figure 3.5). This starvation resistance further supports our hypothesis that ethanol-reared larvae have increased fat storage due to a fatty acid metabolism defect induced by exposure to ethanol during development. 


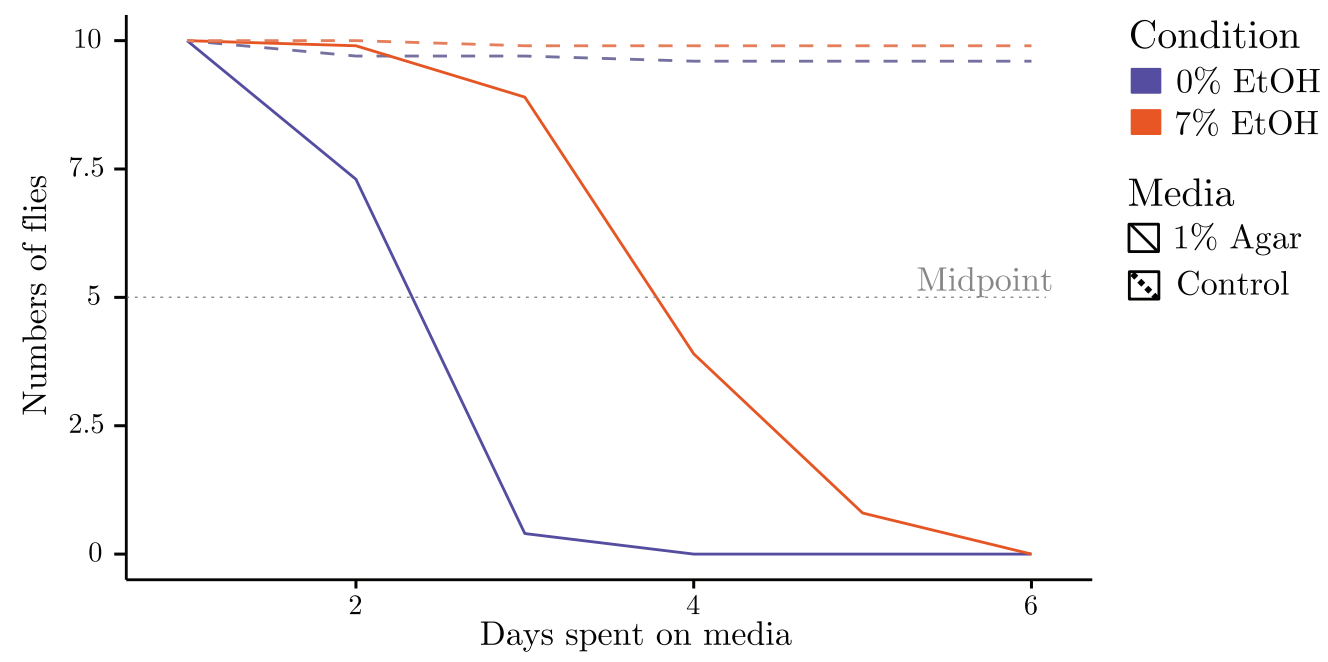

Figure 3.5. Ethanol-reared flies show increased starvation resistance. Adult flies reared in ethanol-containing food show increased resistance to starvation, with median survival of an additional 1.5 days on $1 \%$ agarose medium. Median survival of control flies: 2.3 days; median survival of ethanol-reared flies: 3.8 days. $n=10 . p<0.0001$ (Student's $t$-test).

\section{Interaction Between Saturated Fatty Acids and Ethanol}

CPT1 is required for transport and metabolism of long-chain fatty acids; further, long-chain fatty acids are toxic to humans with mutations in CPT1. In fact, the primary treatment for CPT1 deficiency in humans is removal of long-chain fatty acids from the diets of patients (Bennett \& Santani, 1993). We therefore decided to test the effects of altering the fat content of the flies' food on survival, in both control and ethanol-containing medium. In particular, if ethanol affects survival by impeding fatty acid metabolism, we would expect to see a synergistic effect of ethanol and increased fatty acid intake on survival. We therefore tested the survival of wild-type and $w h d^{1}$ flies reared in control food, ethanol food, palmitic acid (PA), and a combined food (ethanol and PA). Palmitic acid is a long-chain (C16:0) saturated fatty acid found in palm oil and cocoa butter (Denke, 1994;

Fattore \& Fanelli, 2013). Because ethanol affects fatty acid metabolism, and flies 
lacking CPT1 are both defective in the metabolism of long chain fatty acids and sensitive to DAE, we predicted that a diet high in PA would result in reduced survival, similar to what we see in ethanol-reared flies. Finally, we predicted that the combined effect of ethanol and PA during development would be a synergistic effect on survival.

We predicted that an increase in the concentration of PA in fly food would emulate a state of lipid dysregulation where the flies are unable to metabolize all PA consumed as part of their diet. Thus, these flies were expected to have reduced survival relative to control animals. However, our data show that, contrary to our expectation, palmitic acid alone does not affect the survival of wild-type flies at concentrations up to 30\% (Figure 3.6). In addition, consistent with previous data, the $7 \%$ ethanol treatment shows a significant reduction in survival, with only $43.2 \%$ of the flies reaching adulthood. The most interesting result of this assay, however, is the combined effect of ethanol and PA. As noted above, PA-containing food up to $30 \%$ does not have a substantial impact on survival of wild-type flies - the statistical significance of flies consuming $5 \%$ PA is most likely a sporadic result, seeing as the higher concentration of PA (15\%) has no effect. However, when we combined palmitic acid with ethanol, we saw a dramatic reduction in survival that amplifies the effect of ethanol-treatment. This interaction between ethanol and palmitic acid is synergistic, indicating they have the same molecular target. 


\section{A Control}
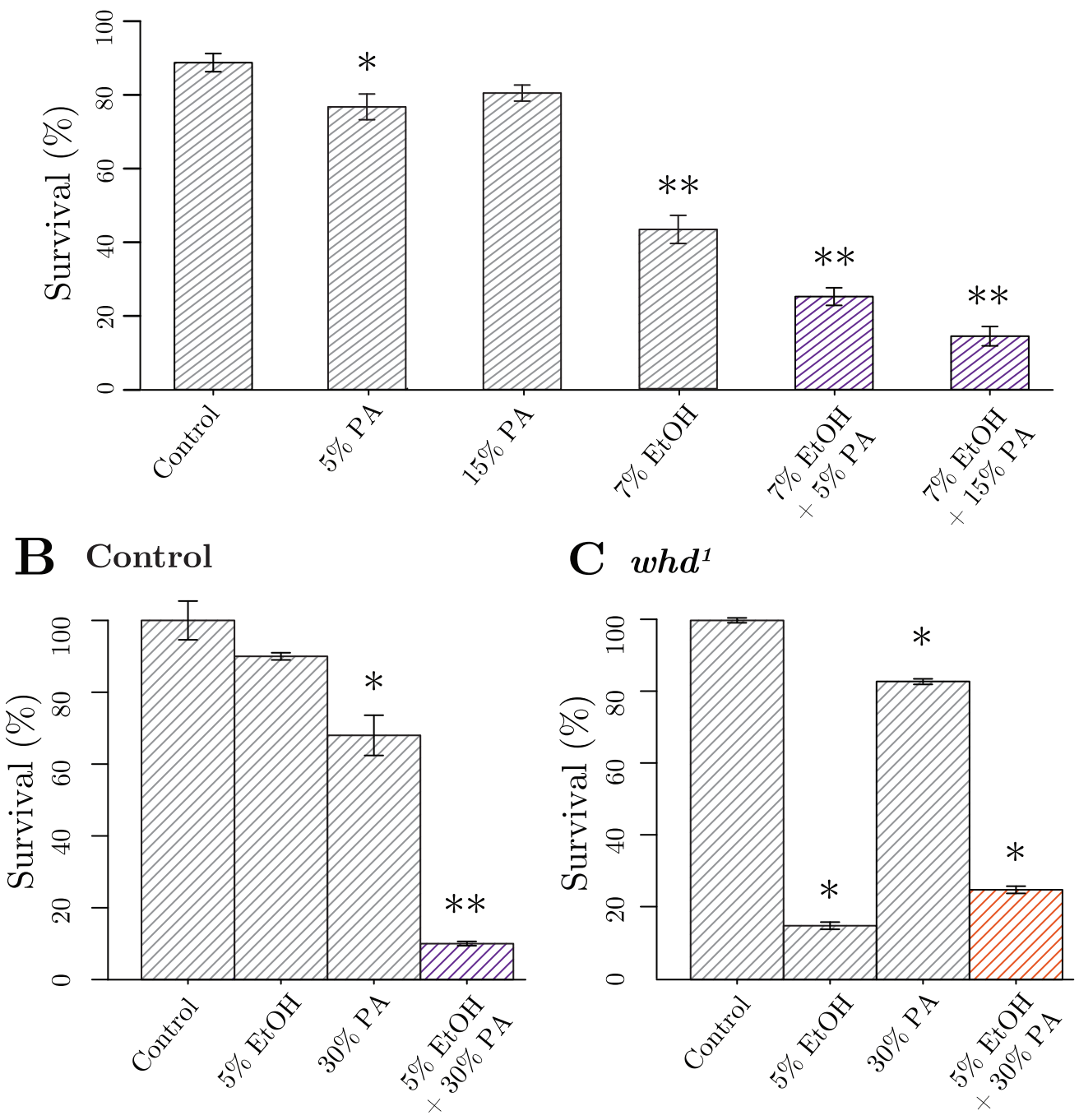

Figure 3.6. Ethanol and palmitic acid have synergistic effect on survival. Flies were reared in food containing $\mathrm{PA}, \mathrm{EtOH}$, combined treatment, or control food. Error bars represent $\pm S E M .{ }^{*} p<0.05,{ }^{* *} p<0.01$ (two-sample $t$-test compared to control). (A) $n=4$, (B-C) $n=2$. 
A two-way ANOVA analysis was performed for Figure 3.6-A to examine the effect of ethanol and palmitic acid on survival (Table 3.3). The analysis confirms a significant interaction between ethanol and palmitic acid $(p=0.007)$. We performed another survival assay, using a sublethal concentration of ethanol and an increased concentration of palmitic acid (Figure 3.6-B). These results confirm our previous data, showing a synergistic effect of ethanol and PA. Thus, our data clearly link developmental alcohol exposure with defects in long-chain fatty acid metabolism.

Table 3.3. Analysis of Variance for Figure 3.6-A

\begin{tabular}{lllcr}
\hline Source & $d f$ & $F$-value & Critical value & $p$-value \\
\hline EtOH & 1 & 534.88 & 3.84 & $<0.0001$ \\
PA & 2 & 22.56 & 5.99 & $<0.0001$ \\
EtOH $\times$ PA & 2 & 6.76 & 5.99 & 0.0069 \\
Error & 17 & & & \\
Total & 22 & & & \\
\hline
\end{tabular}

Note. Two-way ANOVA analysis of Figure 3.6-A. Ethanol and palmitic acid show a very significant interaction that effect the survival of the flies.

Finally, in addition to the observed synergistic effect in wild-type files, $w h d^{1}$ flies display a pronounced sensitivity to $5 \%$ ethanol, and possibly a slight improvement when PA is added (Figure 3.6-C). Because of the profound ethanol sensitivity of the null allele $w h d^{1}$, there is a "floor effect" precluding the easy identification of a synergistic effect of PA and ethanol in this experiment. This could be addressed using hypomorphic alleles of whd that are less sensitive to DAE (see Figure 3.1).

\section{Combined Effect of Sugars and Ethanol}

Sucrose, or sugars in general, are carbohydrates that can be used as a source of energy, converted into glycogen, or used for the synthesis of triglycerides (Gibson, 
Gunn, Wittekind, \& Cottrell, 2013; Schaefer, Gleason, \& Dansinger, 2009). They influence insulin and lipid metabolism (Gibson et al., 2013), elevate blood lipid concentrations, and when available in excess, can be synthesized into fatty acids (Frayn et al., 1995) or converted to fat storage (Hill \& Prentice, 1995). These alterations in lipid metabolism have shown to induce oxidative stress through fat accumulation (Furukawa et al., 2004; Logan-Garbisch et al., 2014).

Additionally, DAE reduces DILP signaling in adult Drosophila, which then leads to an increase in circulating triglycerides as well as increased lipid storage in the fat body (Broughton et al., 2005; Hwangbo et al., 2004; Pospisilik et al., 2010). This dysregulation is phenotypically observable in larvae as obesity, which, in mammals, has been shown to induce oxidative stress (Fernández-Sánchez et al., 2011; Furukawa et al., 2004). Flies fed a high-sugar diet exhibit insulin-resistance and have increased lipid storage and circulating lipids. More importantly, the expression of acetyl-CoA carboxylase (ACC) in these flies is greatly increased (Pasco \& Léopold, 2012). This enzyme plays an essential role in lipogenesis, converting acetyl-CoA into malonyl-CoA, an inhibitor of CPT1 (Rasmussen et al., 2002). If the accumulated fat reduces survival via increased oxidative stress, we predict a drop in survival when flies are reared in food containing an elevated concentration of sugar, similar to the high-fat diet assay (see Figure 3.6).

We tested the survival of flies reared in food with increased sugar, as well as the interaction between increased sugar and ethanol (Figure 3.7). We found that increasing the sugar concentration of the fly food to $8 \%$ did not affect the survival of the flies, nor did adding $8 \%$ sugar to food containing $7 \%$ ethanol enhance the detrimental effects of ethanol in either wild-type or $w h d^{1}$ flies. Interestingly, $8 \%$ 
sugar appears to have slightly increased the survival of $w h d^{1}$ flies.

A Control

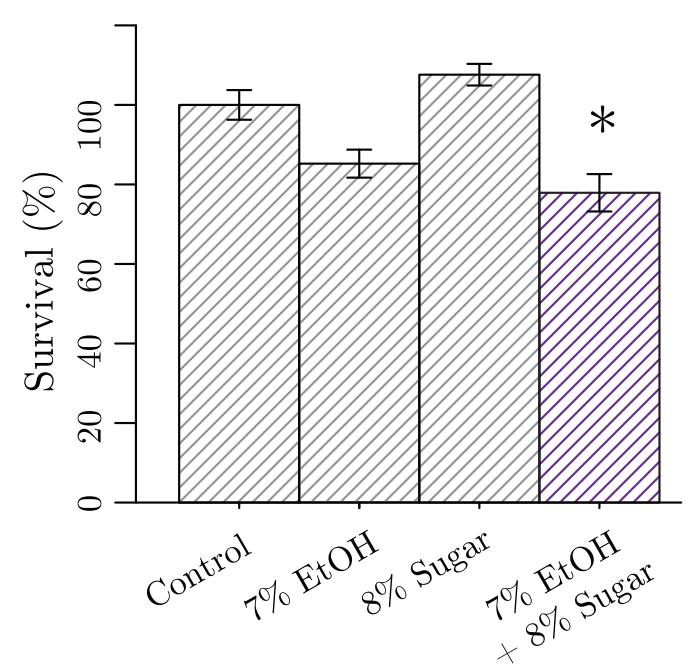

B $w h d^{1}$

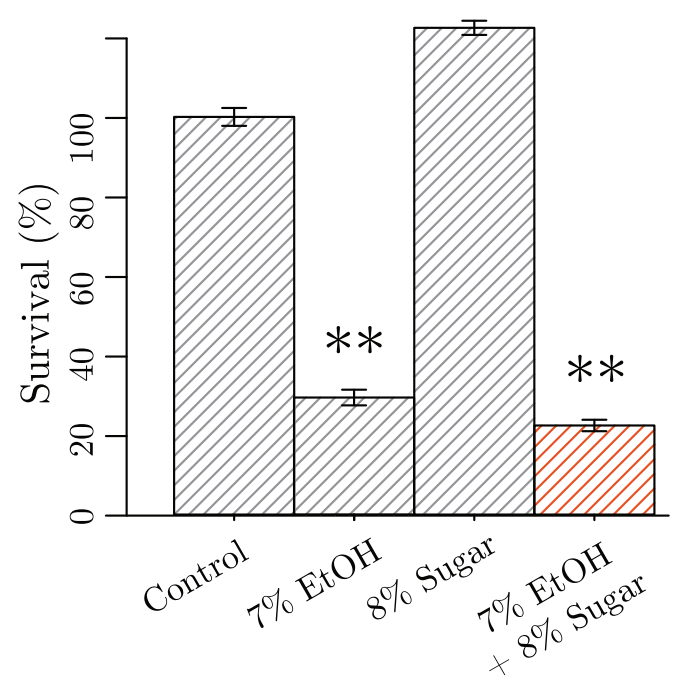

Figure 3.7. Elevated sugar does not reduce survival. Percent survival of control flies (A) and whd $d^{1}$ mutants (B), reared in control food, $7 \%$ ethanol, $8 \%$ sugar, and a combined treatment. Survival has been normalized to genotype-matched control flies reared in control food. $n=4$. Error bars represent $\pm S E M .{ }^{*} p<0.05,{ }^{*} p<0.01$ (two-sample $t$-test).

Elevated sugar levels also had an effect on developmental time of the flies.

The first day of eclosion for wild-type flies on control food was 10 days after egg-laying and 12 days for $8 \%$ sugar food. The first day of eclosion for $w h d^{1}$ files was day 10 and 11 for control and sugar food, respectively. This developmental delay is also present in flies reared in ethanol-containing food. This, coupled with the observed increased survival of flies reared in elevated sugar diet, requires further investigation of the combined effect of sugars and ethanol. Published data indicate that in order to affect survival, we would need to increase the concentration of sugar in the food, to perhaps as high as $20-30 \%$. 


\section{whd Gene Expression in Flies Reared in Ethanol Food}

The sensitivity of $w h d$ flies to ethanol can be explained in at least two ways.

First, whd flies may simply be sensitive to the increased fat storage and consequent increased oxidative stress that results from rearing flies in ethanol. Alternatively, whd itself may be a target of ethanol during development. To distinguish between these hypotheses, we used quantitative real-time PCR (qPCR) to examine whd gene expression in third instar larvae reared in control and $7 \%$ ethanol-containing food. Interestingly, we found that whd mRNA exhibits a 5.3-fold increase in expression of flies reared in ethanol-containing food (Figure 3.8).

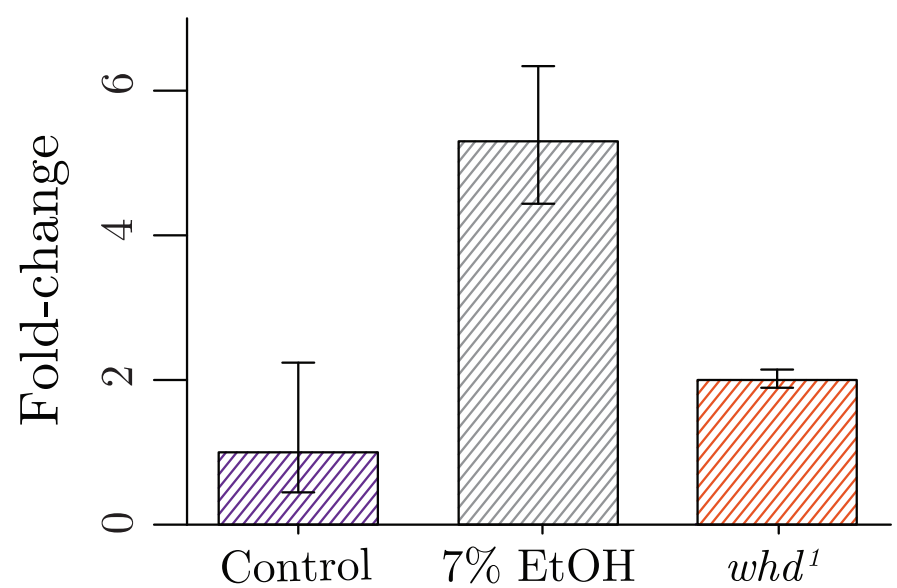

Figure 3.8. whd expression is elevated in wild-type larvae reared in ethanol-containing food. Quantitative RT-PCR results showing a 5.3-fold increase in expression of whd mRNA in ethanol-reared wild-type flies.

This is a surprising result because we predicted reduced or unchanged expression of whd in ethanol-reared larvae. Increased expression might be explained as a cellular mechanism to compensate for the accumulated fat in the ethanol-reared animals. In this case, the ethanol-sensitivity of whd flies would be explained by their inability to fully initiate this compensatory defense mechanism. 
Future experiments will focus on the potential role of whd upregulation as a response to ethanol toxicity. 


\section{Discussion}

DAE causes a wide array of deleterious effects, including low birth weight, increased fetal mortality, developmental delays, craniofacial dysmorphologies, neurobehavioral abnormalities, and moderate to severe intellectual disabilities (Hanson, Jones, \& Smith, 1976; Kodituwakku, 2007; Streissguth et al., 2004). However, despite this understanding that DAE is a powerful teratogen, the mechanisms underlying the myriad of potential phenotypes are not well-understood. This is, in part, because the model systems that best simulate the effects of DAE in humans (vertebrate systems including mice and Xenopus) (Allan, Chynoweth, Tyler, \& Caldwell, 2003; Yelin et al., 2005) are generally not amenable to forward genetic analysis, making unbiased identification of ethanol's targets difficult. We have established a Drosophila model of DAE to begin to uncover additional developmental targets of ethanol. Previously, we showed that the lethality and developmental delay caused by ethanol exposure are largely due to oxidative stress (Logan-Garbisch et al., 2014). In addition, lipid droplet accumulation correlated with increased ROS production (Liu et al., 2015) and high-fat diets in mice increase oxidative stress (Marczuk-Krynicka, Hryniewiecki, Paluszak, Krauss, \& Nowak, 2009). Here, we show that ethanol induces oxidative stress by disrupting long-chain fatty acid metabolism.

\section{Ethanol Induces Oxidative Stress by Disturbing LCFA Metabolism}

whd encodes the enzyme that catalyzes the conversion of long-chain fatty acyl-CoA into acylcarnitine, which is responsible for the transfer of long-chain fatty acids into the mitochondria (Figure 1.1). This allows the utilization of LCFA as an energy source. From a large-scale genetic screen, we identified whd 
as a possible target of DAE. Here, we used two alleles of whd to confirm those results by exposing whd mutant flies to ethanol as they developed (Figure 2.1).

Both $w h d^{1}$ and $w h d^{K G 01596}$ display reduced survival when reared in ethanol-containing food (Figure 3.1). wh $d^{1}$ shows much greater lethality with only $10 \%$ survival on ethanol-containing food. In addition to decreased survival, whd $d^{1}$ flies also display an increase in developmental time when reared in ethanol-containing food (Figure 3.2). The sensitivity and the developmental delay observed with DAE whd flies reveal a connection between DAE's disruption of long-chain fatty acid metabolism and the oxidative stress induced by ethanol exposure.

\section{Ethanol-Reared Flies Have Altered whd Gene Expression}

We observed upregulation in expression of the whd gene in wild-type flies reared in ethanol-containing food (Figure 3.8). We also see a modest upregulation of whd in $w h d^{1}$ flies. It is important to note that $w h d^{1}$, while it is a null mutation, nonetheless produces a nearly full-length transcript (Strub et al., 2008). These data indicate that DAE disrupts long-chain fatty acid metabolism and that the cell may attempt to compensate by the upregulation of whd and other antioxidant genes (Logan-Garbisch et al., 2014). This, combined with the data from Figure 3.1, confirms that some of the oxidative stress induced by ethanol during development is caused by disruption of fatty acid metabolism.

\section{Oxidative Stress Effects Longevity}

Looking at longevity of the flies, we observed that rearing them in ethanol does not impact their average lifespan (Figure 3.3). wh $d^{1}$ flies survive just as long as their wild-type and control-treatment counterparts. However, we saw 
a fifteen day increase in the average lifespan of $w h d^{1}$ flies that were reared in ethanol-containing food compared to those reared in control food. This result was surprising because we were expecting to see the ethanol-exposed $w h d^{1}$ flies to die off earlier. We thought the increased oxidative stress induced by ethanol would reduce the average lifespan of the flies, but the opposite effect was observed. We hypothesize that this phenomenon is caused by activation or upregulation of other antioxidant genes such as Cat, Pxd, and sod1, and that the overexpression of these antioxidant genes in ethanol-reared $w h d^{1}$ flies help increase their average lifespan.

\section{Increased Fat Storage in EtOH-Reared Flies}

Using confocal microscopy, we observed an increase in lipid storage in Nile Red-stained fat bodies of wild-type larvae reared in ethanol-containing food, as well as in $w h d^{1}$ larvae reared in control food (Table 3.2). In wild-type flies reared in ethanol-containing food (Figure 3.4-B), the lipid droplets are increased in both number and size compared to the control (Figure 3.4-A). We see the same size increase in the lipid droplet of $w h d^{1}$ flies (Figure 3.4-D) relative to its wild-type control (Figure 3.4-C).

The wild-type flies reared in ethanol-containing food show the same lipid droplet phenotype as the $w h d^{1}$ flies that lack the enzyme to utilize LCFA. Thus, the increased fat storage observed in ethanol-reared flies is due to lipid metabolism dysfunction, which supports our hypothesis that developmental alcohol exposure leads to lipid accumulation. 


\section{DAE Induces Starvation Resistance}

We further examined lipid accumulation by placing adult flies on media containing $1 \%$ agar, which serves as a source of water without any nutrients. When starved, the median survival of flies that were reared in normal food was 2.3 days (Figure 3.5). In contrast, when we starved ethanol-reared adult flies, their median survival was increased by 1.5 days. We know that DAE induces lipid accumulation, so this starvation resistance is likely explained by the increased fat storage observed in Figure 3.4.

\section{Synergistic Effect of EtOH and Fatty Acids}

Finally, we saw that increasing the concentration of LCFA in fly food did not have a significant effect on survival of wild-type flies (Figure 3.6 A-B). However, when increased LCFAs were combined with ethanol, we observed a significant drop in survival that was more potent than either treatment alone. This shows that the combined treatment of ethanol and palmitic acid are synergistic, strongly suggesting they have the same molecular target.

Since consuming excess amounts of sugar or fats lead to the same outcome (fat buildup), we expected to see the same effect when we reared flies in elevated sugar food. However, the synergistic effect was not observed when we reared wild-type flies in elevated sugar diet and ethanol-containing food (Figure 3.7). Sugar treatment, regardless of ethanol, did not affect survival in either genotype. This is possibly due to the low concentration of sugar used and we address this in Future Directions.

The $w h d^{1}$ flies in these assay exhibited a "floor effect", meaning increasing the treatment concentration did not increase its effect. Due to this diminishing 
effectiveness of $w h d^{1}$ null mutants, we were unable to observe the predicted synergistic interaction. In future experiments, we will use other hypomorphic alleles of whd (see Future Directions).

\section{Future Directions}

To address the "floor effect" seen in $w h d^{1}$ flies (complete loss-of-function mutation), we will perform our dietary assays on flies carrying partial loss-of-function whd mutations. The $w h d^{K G 01596}$ line used in Figure 3.1 is a good candidate for these assays as it has some functional CPT1 enzyme, and does not display the hypersensitivity to ethanol seen in $w h d^{1}$ flies. These experiments will allow us to assay elevated-sugar and high-fat diet to confirm our hypothesis about ethanol's synergistic interaction with sugar and palmitic acid.

We are also performing experiments with higher sugar concentrations. In their study, Musselman et al. (2011) induce hyperglycemia in flies by creating a high-sugar diet that had $5 \times$ the amount of sugar that we used in our assays. They also saw accumulated stored fat, insulin-resistance, and altered transcriptional control of fat and carbohydrate metabolism. With only $8 \%$ sugar increase, flies did not show any decrease in survival and instead, we saw flies do better on this treatment. Higher caloric intake and abundance of readily available energy in the form of sucrose could explain this observation. Since sugars can be converted to fats (Schaefer et al., 2009), we hypothesize that feeding excess sugar would result in the same reduced survival phenotype as excess fatty acid assay due to oxidative stress.

Finally, to confirm our hypothesis about increased antioxidant gene expression in $w h d^{1}$ flies reared in ethanol-containing food, we will examine the expression of 
the antioxidant genes $C a t, P x d$, and sod1. We expect to see an increase in their activity for $w h d^{1}$ flies reared in ethanol-containing food compared to those reared in control food. This would explain the increased longevity of ethanol-reared whd $d^{1}$ we observed in Figure 3.3.

In this study, we learned that developmental alcohol exposure disrupts long-chain fatty acid metabolism, which induces oxidative stress. Flies that were reared in ethanol-containing food were starvation resistant and upregulated whd - the gene encoding an enzyme that catalyzes the transfer of long-chain fatty acids into the mitochondria. We believe this upregulation is a defense mechanism in response to the accumulated fat, which is induced by exposure to ethanol during development. Increased fat storage allows the flies to live longer when starved. Finally, we observed a novel synergistic interaction between ethanol and palmitic acid, which strongly suggests that they both have the same molecular target in the cell. Our results show that one of the mechanisms by which ethanol induces oxidative stress is via dysregulation of fatty acid metabolism. In the long term, we hope to use these results to begin to formulate treatments to mitigate some of the harmful effects of ethanol on the developing fetus. 


\section{References}

Adams, M. D., Celniker, S. E., Holt, R. A., Evans, C. A., Gocayne, J. D., Amanatides, P. G., ... Venter, J. C. (2000). The genome sequence of Drosophila melanogaster. Science, 287, 2185-2195.

Adibhatla, R. M., \& Hatcher, J. F. (2007). Role of lipids in brain injury and diseases. Future Lipidology, 2, 403-422.

Allan, A. M., Chynoweth, J., Tyler, L. A., \& Caldwell, K. K. (2003). A mouse model of prenatal ethanol exposure using a voluntary drinking paradigm. Alcoholism, Clinical and Experimental Research, 27, 2009-2016.

Bennett, M. J., \& Santani, A. B. (1993). Carnitine Palmitoyltransferase $1 A$ Deficiency. Retrieved from http://www.ncbi.nlm.nih.gov/pubmed/20301700

Birse, R. T., Choi, J., Reardon, K., Rodriguez, J., Graham, S., Diop, S., ... Oldham, S. (2010). High-fat-diet-induced obesity and heart dysfunction are regulated by the TOR pathway in Drosophila. Cell Metabolism, 12, 533-544.

Broughton, S. J., Piper, M. D. W., Ikeya, T., Bass, T. M., Jacobson, J., Driege, Y., ... Partridge, L. (2005). Longer lifespan, altered metabolism, and stress resistance in Drosophila from ablation of cells making insulin-like ligands. Proceedings of the National Academy of Sciences of the United States of America, 102, 3105-3110.

Cabiscol, E., Tamarit, J., \& Ros, J. (2000). Oxidative stress in bacteria and protein damage by reactive oxygen species. International Microbiology, 3 , 3-8.

Centers for Disease Control and Prevention. (2012). Alcohol use and binge drinking among women of childbearing age. Retrieved from http://www.cdc.gov/mmwr/preview/mmwrhtml/ mm6128a4.htm?s_cid $=$ mm6128a4_x/

Centers for Disease Control and Prevention. (2014). Fetal alcohol spectrum disorders. Retrieved from http://www.cdc.gov/ncbddd/fasd/diagnosis.html

Chen, J. J., Schenker, S., \& Henderson, G. I. (1997). 4-hydroxynonenal levels are enhanced in fetal liver mitochondria by in utero ethanol exposure. 
Hepatology, 25, 142-147.

Cooke, M. S., Cooke, M. S., Evans, M. D., Evans, M. D., Dizdaroglu, M., Dizdaroglu, M., ... Lunec, J. (2003). Oxidative DNA damage: Mechanisms, mutation, and disease. The FASEB Journal, 17, 1195-1214.

Denke, M. A. (1994). Effects of cocoa butter on serum lipids in humans. American Journal of Clinical Nutrition, 60, 1014S-1016S.

Eaton, S. (2002). Control of mitochondrial beta-oxidation flux. Progress in Lipid Research, 41, 197-239.

Edenberg, H. J., \& Foroud, T. (2013). Genetics and alcoholism. Nature Reviews Gastroenterology \& Hepatology, 10, 487-494.

Fattore, E., \& Fanelli, R. (2013). Palm oil and palmitic acid: A review on cardiovascular effects and carcinogenicity. International Journal of Food Sciences and Nutrition, 64, 648-659.

Fernández-Sánchez, A., Madrigal-Santillán, E., Bautista, M., Esquivel-Soto, J., Morales-González, A., Esquivel-Chirino, C., ... Morales-González, J. a. (2011). Inflammation, oxidative stress, and obesity. International Journal of Molecular Sciences, 12, 3117-3132.

FlyBase. (2015). Flybase allele report: Dmel-whd/kg01596]. Retrieved from http://flybase.org/reports/FBal0134287.html

Frayn, K. N., Kingman, S. M., Sherman, W. M., Khan, S., Wolever, McDonald, R. B., \& Dreher, M. (1995). Dietary sugars and lipid metabolism in humans. American Journal of Clinical Nutrition, 62, 250S-261S.

Furukawa, S., Fujita, T., Shimabukuro, M., Iwaki, M., Yamada, Y., Nakajima, Y., ... Shimomura, I. (2004). Increased oxidative stress in obesity and its impact on metabolic syndrome. Journal of Clinical Investigation, 114, 1752-1761.

Gibson, S., Gunn, P., Wittekind, A., \& Cottrell, R. (2013). The effects of sucrose on metabolic health: A systematic review of human intervention studies in healthy adults. Critical Reviews in Food Science and Nutrition, 53, 591-614. 
Gobin, S., Thuillier, L., Jogl, G., Faye, A., Tong, L., Chi, M., ... Prip-Buus, C. (2003). Functional and structural basis of carnitine palmitoyltransferase 1A deficiency. Journal of Biological Chemistry, 278, 50428-50434.

Gupta, S. C., Hevia, D., Patchva, S., Park, B., Koh, W., \& Aggarwal, B. B. (2012). UPsides and downsides of reactive oxygen species for cancer: The roles of reactive oxygen species in tumorigenesis, prevention, and therapy. Antioxidants \& Redox Signaling, 16, 1295-1322.

Hanson, J. W., Jones, K. L., \& Smith, D. W. (1976). Fetal alcohol syndrome. The Journal of the American Medical Association, 235, 1458-1460.

Heaton, M. B., Paiva, M., Mayer, J., \& Miller, R. (2002). Ethanol-mediated generation of reactive oxygen species in developing rat cerebellum. Neuroscience Letters, 334, 83-86.

Hill, J. O., \& Prentice, A. M. (1995). Sugar and body weight regulation. The American Journal of Clinical Nutrition, 62, 264S-273S.

Howard, R. J., Slesinger, P. a., Davies, D. L., Das, J., Trudell, J. R., \& Harris, R. A. (2011). Alcohol-binding sites in distinct brain proteins: The quest for atomic level resolution. Alcoholism: Clinical and Experimental Research, 35, 1561-1573.

Hwangbo, D. S., Gershman, B., Tu, M.-P., Palmer, M., \& Tatar, M. (2004). Drosophila dFOXO controls lifespan and regulates insulin signalling in brain and fat body. Nature, 429, 562-566.

Jones, K., \& Smith, D. (1973). Recognition of the fetal alcohol syndrome in early infancy. The Lancet, 302, 999-1001.

Juliet Arockia Rani, P., \& Panneerselvam, C. (2001). Carnitine as a free radical scavenger in aging. Experimental Gerontology, 36, 1713-1726.

Kamat, J. P., Devasagayam, T. P., Priyadarsini, K. I., \& Mohan, H. (2000). Reactive oxygen species mediated membrane damage induced by fullerene derivatives and its possible biological implications. Toxicology, 155, 55-61.

Kerner, J., \& Hoppel, C. (2000). Fatty acid import into mitochondria. Biochimica et Biophysica Acta - Molecular and Cell Biology of Lipids, 1486, 1-17. 
Kodituwakku, P. W. (2007). Defining the behavioral phenotype in children with fetal alcohol spectrum disorders. Neuroscience \& Biobehavioral Reviews, 31, 192-201.

Kohen, R., \& Nyska, A. (2002). Oxidation of biological systems: Oxidative stress phenomena, antioxidants, redox reactions, and methods for their quantication. Toxicologic Pathology, 30, 620-650.

Kregel, K. C., \& Zhang, H. J. (2007). An integrated view of oxidative stress in aging: Basic mechanisms, functional effects, and pathological considerations. American Journal of Physiology, 292, R18-R36.

Listenberger, L. L., Han, X., Lewis, S. E., Cases, S., Farese, R. V., Ory, D. S., \& Schaffer, J. E. (2003). Triglyceride accumulation protects against fatty acid-induced lipotoxicity. Proceedings of the National Academy of Sciences of the United States of America, 100, 3077-3082.

Liu, L., Zhang, K., Sandoval, H., Yamamoto, S., Jaiswal, M., Sanz, E., ... Bellen, H. J. (2015). Article glial lipid droplets and ROS induced by mitochondrial defects promote neurodegeneration. Cell, 160, 177-190.

Logan-Garbisch, T., Bortolazzo, A., Luu, P., Ford, A., Do, D., Khodabakhshi, P., \& French, R. L. (2014). Developmental ethanol exposure leads to dysregulation of lipid metabolism and oxidative stress in Drosophila. G3: Genes/Genomes/Genetics, 5, 49-59.

Lupton, C., Burd, L., \& Harwood, R. (2004). Cost of fetal alcohol spectrum disorders. American Journal of Medical Genetics, 127C, 42-50.

Mahuran, D. J. (1999). Biochemical consequences of mutations causing the GM2 gangliosidoses. Biochimica et Biophysica Acta - Molecular Basis of Disease, 1455, 105-138.

Marczuk-Krynicka, D., Hryniewiecki, T., Paluszak, J., Krauss, H., \& Nowak, D. (2009). High fat content in diets and oxidative stress in livers of non-diabetic and diabetic rats. Polish Journal of Environmental Studies, 18, 249-253.

Massaad, C., \& Klann, E. (2011). Reactive oxygen species in the regulation of synaptic plasticity and memory. Antioxidants 83 Redox Signaling, 14, 2013-2054. 
McClure, K. D., French, R. L., \& Heberlein, U. (2011). A Drosophila model for fetal alcohol syndrome disorders: Role for the insulin pathway. Disease Models \&3 Mechanisms, 4, 335-346.

Miranda, S. R., He, X., Simonaro, C. M., Gatt, S., Dagan, A., Desnick, R. J., \& Schuchman, E. H. (2000). Infusion of recombinant human acid sphingomyelinase into niemann-pick disease mice leads to visceral, but not neurological, correction of the pathophysiology. The FASEB Journal, 14, 1988-1995.

Mittal, M., Siddiqui, M. R., Tran, K., Reddy, S. P., \& Malik, A. B. (2014). Reactive oxygen species in inflammation and tissue injury. Antioxidants 8 Redox Signaling, 20, 1126-1167.

Mukherjee, S., \& Maxfield, F. R. (2004). Lipid and cholesterol trafficking in NPC. Biochimica et Biophysica Acta - Molecular and Cell Biology of Lipids, 1685, 28-37.

Musselman, L. P., Fink, J. L., Narzinski, K., Ramachandran, P. V., Hathiramani, S. S., Cagan, R. L., \& Baranski, T. J. (2011). A high-sugar diet produces obesity and insulin resistance in wild-type Drosophila. Disease Models $\mathbb{E}$ Mechanisms, 4, 842-849.

Mylonas, C., \& Kouretas, D. (1999). Lipid peroxidation and tissue damage. In Vivo, 13, 295-309.

National Institutes of Health. (2014). Carnitine palmitoyltransferase 1 deficiency. Retrieved from http://ghr.nlm.nih.gov/condition/carnitine-palmitoyltransferase-i-deficiency

Nguyen, P., Leray, V., Diez, M., Serisier, S., Le Bloc'H, J., Siliart, B., \& Dumon, H. (2008). Liver lipid metabolism. Journal of Animal Physiology and Animal Nutrition, 92, 272-283.

Olpin, S. E., Allen, J., Bonham, J. R., Clark, S., Clayton, P. T., Calvin, J., ... Tanner, M. S. (2001). Features of carnitine palmitoyltransferase type I deficiency. Journal of Inherited Metabolic Disease, 24, 35-42.

Oyinloye, B., Adenowo, A., \& Kappo, A. (2015). Reactive oxygen species, apoptosis, antimicrobial peptides and human inflammatory diseases. 
Pharmaceuticals, 8, 151-175.

Pandey, U. B., \& Nichols, C. D. (2011). Human disease models in Drosophila melanogaster and the role of the fly in therapeutic drug discovery. Drug Delivery, 63, 411-436.

Pasco, M. Y., \& Léopold, P. (2012). High sugar-induced insulin resistance in Drosophila relies on the lipocalin Neural Lazarillo. PLoS ONE, 7, 36583.

Pospisilik, J. A., Schramek, D., Schnidar, H., Cronin, S. J. F., Nehme, N. T., Zhang, X., ... Penninger, J. M. (2010). Drosophila genome-wide obesity screen reveals hedgehog as a determinant of brown versus white adipose cell fate. Cell, 140, 148-160.

Price, N. T., Jackson, V. N., Müller, J., Moffat, K., Matthews, K. L., Orton, T., \& Zammit, V. a. (2010). Alternative exon usage in the single CPT1 gene of Drosophila generates functional diversity in the kinetic properties of the enzyme. The Journal of Biological Chemistry, 285, 7857-7865.

Pulsifer, M. B. (1996). The neuropsychology of mental retardation. Journal of the International Neuropsychological Society, 2, 159-176.

Ramachandran, V., Watts, L. T., Maffi, S. K., Chen, J., Schenker, S., \& Henderson, G. I. (2003). Ethanol-induced oxidative stress precedes mitochondrially mediated apoptotic death of cultured fetal cortical neurons. Journal of Neuroscience Research, 74, 577-588.

Rasmussen, B. B., Holmbäck, U. C., Volpi, E., Morio-Liondore, B., Paddon-Jones, D., \& Wolfe, R. R. (2002). Malonyl coenzyme A and the regulation of functional carnitine palmitoyltransferase- 1 activity and fat oxidation in human skeletal muscle. Journal of Clinical Investigation, 110, 1687-1693.

Salganik, R. I. (2001). The benefits and hazards of antioxidants: Controlling apoptosis and other protective mechanisms in cancer patients and the human population. Journal of the American College of Nutrition, 20, 464-472.

Schaefer, E. J., Gleason, J. A., \& Dansinger, M. L. (2009). Dietary fructose and glucose differentially affect lipid and glucose homeostasis. The Journal of Nutrition, 139, 1257S-1262S. 
Smith, S. M., Garic, A., Berres, M. E., \& Flentke, G. R. (2014). Genomic factors that shape craniofacial outcome and neural crest vulnerability in FASD. Frontiers in Genetics, 5, 224.

Stowe, D. F., \& Camara, A. K. S. (2009). Mitochondrial reactive oxygen species production in excitable cells: Modulators of mitochondrial and cell function. Antioxidants \& Redox Signaling, 11, 1373-1414.

Streissguth, A. P., Bookstein, F. L., Barr, H. M., Sampson, P. D., O'Malley, K., \& Young, J. K. (2004). Risk factors for adverse life outcomes in fetal alcohol syndrome and fetal alcohol effects. Journal of Developmental \& Behavioral Pediatrics, 25, 228-238.

Strub, B. R., Parkes, T. L., Mukai, S. T., Bahadorani, S., Coulthard, A. B., Hall, N., ... Hilliker, A. J. (2008). Mutations of the withered (whd) gene in Drosophila melanogaster confer hypersensitivity to oxidative stress and are lesions of the carnitine palmitoyltransferase I (CPT I) gene. Genome / National Research Council Canada = Genome / Conseil National de Recherches Canada, 51, 409-420.

Sykiotis, G. P., \& Bohmann, D. (2008). Keap1/Nrf2 signaling regulates oxidative stress tolerance and lifespan in Drosophila. Developmental Cell, 14, 76-85.

U.S. Department of Health and Human Services. (2014). Results from the 2013 national survey on drug use and health: Summary of national findings. Retrieved from http://www.samhsa.gov/data/sites/default/files/ NSDUHresultsPDFWHTML2013/Web/NSDUHresults2013.pdf

Valko, M., Leibfritz, D., Moncol, J., Cronin, M. T. D., Mazur, M., \& Telser, J. (2007). Free radicals and antioxidants in normal physiological functions and human disease. The International Journal of Biochemistry \& Cell Biology, $39,44-84$.

Vance, J. E. (2006). Lipid imbalance in the neurological disorder, Niemann-Pick C disease. Federation of European Biochemical Societies Letters, 580, 5518-5524.

van Montfort, R. L. M., Congreve, M., Tisi, D., Carr, R., \& Jhoti, H. (2003). Oxidation state of the active-site cysteine in protein tyrosine phosphatase 1B. Nature, 423, 773-777. 
Varghese, J., Lim, S. F., \& Cohen, S. M. (2010). Drosophila miR-14 regulates insulin production and metabolism through its target, sugarbabe. Genes and Development, 24, 2748-2753.

Wattendorf, D., \& Muenke, M. (2005). Fetal alcohol spectrum disorders. American Family Physician, 72, 279-285.

Wu, D., \& Cederbaum, A. I. (2003). Alcohol, oxidative stress, and free radical damage. Alcohol Research \&3 Health, 27, 277-284.

Yelin, R., Schyr, R. B. H., Kot, H., Zins, S., Frumkin, A., Pillemer, G., \& Fainsod, A. (2005). Ethanol exposure affects gene expression in the embryonic organizer and reduces retinoic acid levels. Developmental Biology, 279, 193-204.

Zou, J., Sweeney, C. L., Chou, B. K., Choi, U., Pan, J., Wang, H., ... Malech, H. L. (2011). Oxidase-deficient neutrophils from X-linked chronic granulomatous disease iPS cells: Functional correction by zinc finger nuclease-mediated safe harbor targeting. Blood, 117, 5561-5572. 